\title{
Mid and Far IR properties of late-type galaxies in the Coma and A1367 clusters: ISOCAM and ISOPHOT observations ${ }^{\star}$
}

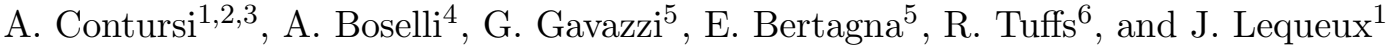 \\ 1 DEMIRM, Observatoire de Paris, 61 Av. de l'Observatoire, 75014 Paris, France \\ 2 Service d'Astrophysique, Centre d'Étude de Saclay, 91191 Gif-sur-Yvette, France \\ 3 IPAC, JPL, Caletch, 100-22, Pasadena, CA 91125, USA \\ 4 Laboratoire d'Astronomie Spatiale, BP 8, Traverse du Siphon, 13376 Marseille, France \\ 5 Universitá di Milano - Bicocca, P.zza dell'Ateneo Nuovo 1, 20126 Milano, Italy \\ ${ }^{6}$ MPI für Kernphysik, Postfach 103980, 69117 Heidelberg, Germany
}

Received 13 December 1999 / Accepted 28 September 2000

\begin{abstract}
We present Mid (MIR) and Far (FIR) Infrared observations of 18 spiral/irregular galaxies belonging to the Coma and A1367 clusters, carried out with the CAM and PHOT instruments on board the ISO satellite. Complementary photometry from the UV to the Near Infrared (NIR) together with $\mathrm{H} \alpha$ imaging, HI and ${ }^{12} \mathrm{CO}$ line measurements allow us to study the relationships between the IR emission and the star formation properties of these galaxies. Most of the resolved galaxies show extended MIR emission throughout their disks even where no $\mathrm{H} \alpha$ emission is present. This suggests that the Aromatic carriers can be excited by the general interstellar radiation field (ISRF), i.e. by visible photons. Only close to HII regions the UV photons are the principal sources of Aromatic carrier excitation. However, when the UV radiation field becomes intense enough these carriers can be destroyed. The average integrated $15 / 6.75 \mu \mathrm{m}$ ratio of the observed galaxies is $\sim 1$, i.e. the typical value for the photodissociation regions (PDRs). This suggests that, despite the high star formation rate (SFR) and the very luminous HII regions of these galaxies, their integrated MIR emission is dominated by PDR-like regions rather than HII-like regions. A cold dust component with average temperature $\sim 22 \mathrm{~K}$ exists in most of the target galaxies, probably arising from big dust grains (BGs) in thermal equilibrium with the ISRF. The contribution to the BGs heating from the ionizing stars decreases with increasing wavelength. A warmer dust component whose emission dominates the spectrum between 20 and $100 \mu \mathrm{m}$ is likely to exist. This is probably due to both Very Small Grains (VSGs) and warm BGs emission. The dust to gas ratio of the target galaxies is comparable to that of the solar neighborhood. There is a weak trend between the dust total mass and both the atomic and molecular gas content. The MIR and FIR properties of the analyzed galaxies do not seem to be affected by the environment despite the fact that most of the targets are interacting with the Intra-Cluster-Medium.
\end{abstract}

Key words. galaxies: clusters: Coma; A 1367 - galaxies: ISM - galaxies: irregular - galaxies: spiral infrared: galaxies

\section{Introduction}

The infrared (5 $\mu \mathrm{m}<\lambda<1 \mathrm{~mm}$ ) emission from normal galaxies arises from dust that thermally re-emits absorbed starlight (Beichman 1987; Soifer et al. 1987). The IRAS observations, coupled with detailed measurements of the extinction curve, allowed to improve our understanding of the composition, formation and the evolution of dust (Mathis 1990; Puget \& Léger 1989). Following

Send offprint requests to: A. Contursi,

e-mail: contursi@ipac.caltech.edu

* Based on observations with ISO, an ESA project with instruments funded by ESA member states (especially the PI countries: France, Germany, The Netherlands and the UK) and with the participation of ISAS and NASA. the model proposed by Désert et al. (1990), three main dust components explain the observations. 1) A big grain (BG) component, whose composition (carbons, silicate or both) is not yet exactly known. Due to their average size of $\simeq 0.1 \mu \mathrm{m}$, these grains can reach thermal equilibrium with the interstellar radiation field (ISRF), with average temperatures ranging from $15 \mathrm{~K}$ up to $30 \mathrm{~K}$ (Draine \& Lee 1984). Thus, the bulk of the emission from these grains is in the Far infrared (FIR) and submillimeter domains. This component is responsible for the linear rise in the visible-near infrared (NIR) part of the extinction curve. 2) To explain the UV rise of the extinction curve $(\lambda>1800 \AA)$, extremely small grains or macro-molecules (50-100 atoms) must be invoked. Moreover, the Mid infrared (MIR, $2 \mu \mathrm{m}<\lambda<20 \mu \mathrm{m}$ ) 
emission spectra of the Galactic and extragalactic interstellar medium show emission bands certainly due to aromatic compounds, most likely Polycyclic Aromatic Hydrocarbons (PAHs) (Allamandola et al. 1989; Léger \& Puget 1984; Puget \& Léger 1989; Allamandola et al. 1999), excited by stochastic heating. For this reason, hereafter, we will refer to these MIR emission features as to the Aromatic Infrared bands (AIBs). One clear-cut result of the Infrared Space Observatory (ISO) observations (Kessler et al. 1996) was to show that AIBs are present in most of the Interstellar Medium's (ISM) phases, (cirrus, PDRs, low ionized medium, surface of molecular clouds) in the Milky Way and in external galaxies (Cesarsky et al. 1996b, 1996a; Boulanger et al. 1996; Laurent et al. 1999; Le Coupanec et al. 1999; Mirabel et al. 1990; Boulade et al. 1996; Helou et al. 2000). 3) BGs and AIBs carriers do not explain the excess at $25 \mu \mathrm{m}$ found by IRAS. It is for this reason that another component has been invoked: the very small grains (VSGs) (Désert et al. 1990). The real nature of these carriers is still unclear. They are probably carbonaceous but with larger sizes $(10-100 \AA)$ than the AIBs carriers. They are transiently heated, but the probability of absorbing another photon before they cool down is greater than for AIB carriers, bringing them closer to thermal equilibrium with the ISRF than are the AIBs carriers. Their emitting properties for a fixed grain radius change as a function of the ISRF: their $\Delta T / T$ gets smaller as the ISRF increases, i.e. their emission spectrum approaches a thermal one for high ISRF (Draine \& Anderson 1985).

An accurate analysis of the physical properties of the ISM can be done only once the heating sources of the dust components are unambiguously established. Devereux \& Young $(1990,1993)$ and Devereux \& Scowen (1994) claim that the FIR emission in nearby normal galaxies is primarily due to dust heated by OB stars. This result was particularly appealing since it gave a star formation rate tracer not affected by extinction. On the other hand, other authors (Helou 1986; Persson \& Helou 1987; Walterbos \& Schwering 1987; Rice et al. 1990; Xu \& Helou 1996) found that the FIR emission in spiral galaxies is due to two components: one quiescent heated primarily by the general ISRF (cirrus component) and the other composed of dust closer to HII regions (active component). The cirrus contribution at FIR wavelengths in normal galaxies can be as high as $70 \%$, depending on the morphological type: its contribution to the total FIR luminosity is higher in early type galaxies than in late-type galaxies (Sauvage \& Thuan 1992). The cirrus component is characterized by lower IRAS $60 / 100$ color temperatures than the active component. This is directly related to the fact that the infrared emission of the big grains in thermal equilibrium with the ISRF shifts systematically towards longer wavelengths as the dust temperature decreases. Thus, for normal galaxies, the peak of the big-grain emission occurs at wavelengths higher than the IRAS $100 \mu \mathrm{m}$ band. Moreover, the IRAS analysis of nearby resolved galaxies showed that the relative contribution of the active and cirrus component in galaxies depends on the relative space distribution of stars and dust. Even if the active component in late type spirals dominates the total FIR emission, the cirrus contribution can be important over the disk.

The environment can play a fundamental role on the dust properties of galaxies. Using SCUBA sub-millimeter observations of the nearby normal galaxy NGC 891 Israel et al. (1999) found a cold dust component at a temperature $\sim 21 \mathrm{~K}$. For the same galaxy Alton et al. (2000) found that, though the bulk of the sub-millimeter emission is associated with the molecular gas, it extends beyond the optical radius, where it follows the distribution of the neutral HI gas. This dust component could be easily removed during the ram pressure stripping process occurring on cluster galaxies. Previous IRAS analyses of cluster galaxies gave ambiguous results: Bicay \& Giovanelli (1987) found that HI deficient and not deficient galaxies have comparable dust masses. Conversely, Doyon \& Joseph (1995) found that galaxies belonging to the Virgo cluster core, are dust-deficient.

To study the relative contribution of the cirrus and active components in spiral galaxies as well as the environmental effects on their dust content, we prepared a coherent program of observations with the ISO satellite aimed at studying the properties of the ISM in various regions in the Milky Way, in nearby resolved galaxies such as the Magellanic Clouds, M 31, M 51, M 101, in a large sample of optically selected galaxies in the Virgo cluster and of star forming galaxies in the Coma supercluster. The present paper reports the ISO observations of 18 late-type galaxies belonging to the Coma and A1367 clusters. The sample includes objects with peculiarities suggesting a recent interaction with the environment, thus it should represent an ideal test-bed to see if the cold dust is somehow perturbed by the galaxy-IGM interaction.

The paper is organized as follows: the sample is presented in Sect. 2, the observations and data reduction in Sect. 3, the results and discussion in Sects. 4 and 5 respectively and the conclusions are summarized in Sect. 6 .

\section{The sample}

We present ISO observations of 18 late-type galaxies that lie within $2^{0}$ or $1^{0}$ projected radial distance from the X-ray centers of the Coma and A1367 clusters, respectively. Most of the observed galaxies are high star forming systems with peculiar or irregular morphology. Fourteen out of eighteen observed galaxies were selected for their blue color indices, i.e. $(B-H) \leq 2.75$, among the cluster members with $\mathrm{S} / \mathrm{Irr}$ morphology previously detected by IRAS at $60 \mu \mathrm{m}$. The remaining 4 targets, which do not satisfy the above criterion, were selected among less actively star-forming late-type members of the two clusters, which nevertheless show interesting structural properties, such as a strong HI deficiency (CGCG 160088, CGCG 160095, CGCG 160260; Sullivan et al. 1981) or asymmetry in their HI (CGCG 160026; Gavazzi et al. 1989) and $\mathrm{H} \alpha$ distributions (CGCG 160026 and 
Table 1. The target galaxies

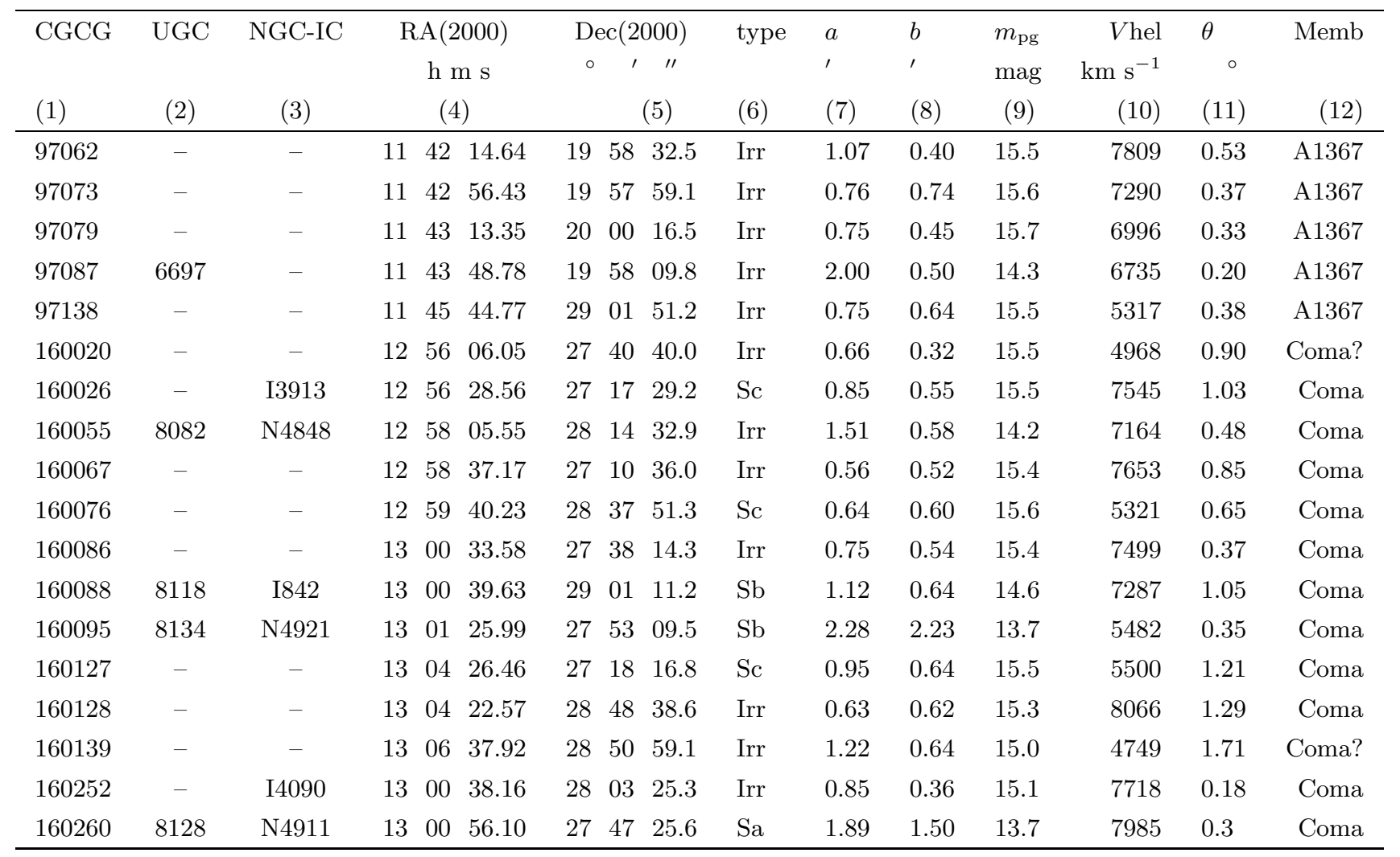

CGCG 160088; Gavazzi et al. 1998) that could suggest a dynamical interaction with the intracluster medium (ICM).

All but two (CGCG 160020 and CGCG 160139) are cluster members according to the criterion given by Gavazzi et al. (1995a): the combination of their recessional velocity and projected distance from the clusters are such that they lie within the "caustics" associated with the cluster potential wells. The memberships of the two remaining objects are uncertain due to their low recessional velocities (4968 and $4749 \mathrm{~km} \mathrm{~s}^{-1}$ respectively). It should be emphasized that, beside the criteria given above, the present sample has not been selected according to an optical completeness criterion.

Table 1 lists the general properties of the target galaxies as follows:

Columns 1, 2 and 3: CGCG (Zwicky 1961-68), UGC (Nilson 1973); and NGC/IC designations;

Columns 4 and 5: celestial coordinates (J2000);

Column 6: morphological types. The Irr designation stands for relatively optical bright $\left(M_{B}<-19\right)$ galaxies with a peculiar morphology;

Columns 7 and 8: major and minor diameters in arcmin at the 25 th $B$ isophotal magnitude;

Column 9: photographic magnitude $m_{\mathrm{p}}$ as reported in the CGCG;

Column 10: heliocentric velocity in $\mathrm{km} \mathrm{s}^{-1}$;
Column 11: projected distances from the respective X-ray centers in degrees;

Column 12: Belonging cluster. The assumed distances for Coma and A1367 are 69 and $65 \mathrm{Mpc}$ respectively $\left(H_{0}=100 \mathrm{~km} \mathrm{~s}^{-1} \mathrm{Mpc}^{-1}\right)$.

Data from the radio centimetric to the UV are also available and they are listed in Table 2.

Column 1: CGCG name;

Columns 2, 3 and 4: IRAS flux in Jy at 100, 60 and $25 \mu \mathrm{m}$ (Bicay \& Giovanelli 1987; Lari, private communication);

Columns 5, 6 and \%: $K, H$ and $J$ magnitudes (Gavazzi \& Boselli 1996);

Columns 8, 9 and 10: $V, B$ and $U$ magnitudes (Gavazzi \& Boselli 1996);

Column 11: UV flux at $2000 \AA$ (Donas et al. 1990, 1995); All but the UV magnitudes are corrected for internal extinction following Gavazzi \& Boselli (1996);

Column 12: $\mathrm{H} \alpha$ Equivalent Width (E.W.) (Gavazzi et al. 1998 and references therein);

Column 13: the logarithm of the $\mathrm{HI}$ mass (in $M_{\odot}$ ) (Gavazzi et al. 1989);

Column 14: the HI deficiency parameter as defined in Giovanelli \& Haynes (1985);

Column 15: the logarithm of the $M_{\mathrm{H}_{2}}$ mass (in $M_{\odot}$ ). $\mathrm{H}_{2}$ masses were derived from $\mathrm{CO}(1-0)$ line measurements assuming, as in Boselli et al. (1997b), a CO- $\mathrm{H}_{2}$ conversion factor $X$ :

$X=2.310^{20} \mathrm{~mol} \mathrm{~cm}{ }^{-2}\left(\mathrm{~K} \mathrm{~km} \mathrm{~s}^{-1}\right)^{-1}$. 
Table 2. Photometric parameters of the target galaxies

\begin{tabular}{|c|c|c|c|c|c|c|c|c|c|c|c|c|c|c|}
\hline CGCG & $\begin{array}{c}100 \mu \mathrm{m} \\
\mathrm{Jy} \\
(2) \\
\end{array}$ & $\begin{array}{r}60 \mu \mathrm{m} \\
\mathrm{Jy} \\
(3) \\
\end{array}$ & $\begin{array}{r}25 \mu \mathrm{m} \\
\mathrm{Jy} \\
(4) \\
\end{array}$ & $\begin{array}{r}K \\
\text { mag } \\
(5) \\
\end{array}$ & $\begin{array}{r}H \\
\text { mag } \\
(6) \\
\end{array}$ & $\begin{array}{r}J \\
\text { mag } \\
(7) \\
\end{array}$ & $\begin{array}{r}V \\
\text { mag } \\
(8) \\
\end{array}$ & $\begin{array}{r}B \\
\text { mag } \\
(9) \\
\end{array}$ & $\begin{array}{r}U \\
\text { mag } \\
(10) \\
\end{array}$ & $\begin{array}{l}U V \\
\text { mag } \\
(11) \\
\end{array}$ & $\begin{array}{r}\mathrm{H} \alpha \text { E.W. } \\
\AA \\
(12) \\
\end{array}$ & $\begin{array}{c}\log M_{\mathrm{HI}} \\
M_{\odot} \\
(13)\end{array}$ & $D e f_{\mathrm{HI}}$ & $\begin{array}{r}\log M_{\mathrm{H}_{2}} \\
M_{\odot} \\
(15) \\
\end{array}$ \\
\hline 97062 & $<0.37$ & 0.28 & $<0.14$ & - & 12.65 & - & 14.42 & 14.71 & 14.62 & 15.00 & 49 & 9.03 & 0.41 & $<8.39$ \\
\hline 97073 & $<0.37$ & 0.20 & $<0.14$ & 12.98 & 13.28 & 13.92 & 15.32 & 15.71 & 15.57 & 14.60 & 94 & 9.02 & 0.24 & 8.81 \\
\hline 97079 & 0.65 & 0.39 & $<0.14$ & 13.24 & 13.40 & 14.04 & 15.04 & 15.41 & 14.89 & 14.20 & 137 & 8.82 & 0.35 & 8.70 \\
\hline 97087 & 3.41 & 1.58 & 0.28 & 10.54 & 10.72 & 11.39 & 12.60 & 12.90 & 12.43 & 12.70 & 74 & 9.54 & 0.29 & 9.53 \\
\hline 97138 & $<0.37$ & 0.22 & $<0.14$ & - & 13.41 & - & 14.98 & 15.38 & 15.20 & 14.20 & 64 & 9.39 & -0.12 & $<8.20$ \\
\hline 160020 & 1.05 & 0.65 & $<0.11$ & - & 12.98 & - & 14.71 & 14.94 & - & 14.90 & 35 & 8.64 & 0.59 & 8.46 \\
\hline 160026 & 0.44 & 0.26 & $<0.11$ & - & 12.54 & - & 14.83 & 15.42 & 15.37 & 15.30 & 33 & 9.00 & 0.25 & 8.61 \\
\hline 160055 & 2.75 & 1.40 & 0.30 & 10.40 & 10.78 & 11.29 & 13.22 & 13.75 & 13.66 & 13.80 & 34 & 9.05 & 0.55 & 9.19 \\
\hline 160067 & 0.83 & 0.45 & $<0.11$ & - & 13.02 & - & 15.16 & 15.49 & 15.27 & 14.70 & 75 & 9.09 & 0.05 & 8.87 \\
\hline 160076 & 0.17 & 0.13 & $<0.14$ & - & 13.63 & - & 15.42 & 15.74 & 15.54 & 14.60 & 47 & 9.35 & -0.34 & - \\
\hline 160086 & 0.46 & 0.15 & $<0.11$ & 13.14 & 13.16 & 14.05 & 15.13 & 15.45 & 15.20 & 14.90 & 39 & 8.45 & 0.86 & 7.86 \\
\hline 160088 & 0.88 & 0.25 & 0.21 & - & 11.06 & - & 13.67 & 14.33 & 14.54 & 15.24 & 15 & 9.07 & 0.47 & 9.10 \\
\hline 160095 & 0.67 & 0.23 & $<0.14$ & 9.26 & 9.46 & 10.21 & 12.50 & 13.42 & 13.94 & 14.90 & 4 & 9.04 & 0.85 & 9.29 \\
\hline 160127 & 0.44 & 0.23 & $<0.11$ & - & 13.27 & - & 14.82 & 15.23 & 15.04 & - & 68 & 9.42 & -0.08 & $<8.38$ \\
\hline 160128 & 0.44 & 0.22 & $<0.11$ & - & 13.50 & - & 15.17 & 15.47 & - & 14.10 & 79 & 9.31 & -0.25 & 8.60 \\
\hline 160139 & 0.58 & 0.36 & $<0.11$ & - & 13.07 & - & 14.16 & 14.61 & 14.17 & 13.97 & 53 & 9.67 & -0.09 & 8.32 \\
\hline 160252 & 2.64 & 1.38 & 0.15 & 11.62 & 12.08 & 12.62 & 14.04 & 14.52 & 14.17 & 14.80 & 35 & 8.72 & 0.66 & 9.13 \\
\hline 160260 & 2.46 & 0.78 & $<0.11$ & 9.82 & 10.02 & 10.79 & 12.70 & 13.48 & 13.83 & 14.70 & 8 & 8.90 & 0.87 & 9.44 \\
\hline
\end{tabular}

\section{ISO observations and data reduction}

\subsection{ISOCAM data}

ISOCAM (Cesarsky et al. 1996) observations were taken in June 1996, through the two broad band filters LW2 $(5-8.5 \mu \mathrm{m})$ and LW3 $(12-18 \mu \mathrm{m})$, with a pixel size of 6 " $/$ pixel. The effective angular resolution is approximately $6^{\prime \prime}$ and $8^{\prime \prime}$ for LW2 and LW3, respectively. Images are mosaics of $2 \times 2$ raster positions obtained in the microscanning mode with shifts of 10 pixels $\left(60^{\prime \prime}\right)$ along both $x$ and $y$ directions. The final image size is of $42 \times 42$ pixels, corresponding to a total field of view of $4.2^{\prime} \times 4.2^{\prime}$. The central $22 \times 22$ pixels of the final image, containing the targets, were sampled 4 times. Three galaxies (CGCG 160055, CGCG 160095 and CGCG 160252) were re-observed in July 1997. CGCG 160055 and CGCG 160252 were observed with the LW2 filter using a pixel size equal to $3^{\prime \prime} /$ pixel. CGCG 160095 was observed with both the LW2 and LW3 filters and $6^{\prime \prime} /$ pixel size mode. These images are mosaics of $4 \times 4$ raster positions obtained in microscanning mode with shifts of 8 pixels along both $x$ and $y$ directions. Thus, the total fields of view is equal to $2.8^{\prime} \times 2.8^{\prime}$ for CGCG 160055 and CGCG 160252 and $5.6^{\prime} \times 5.6^{\prime}$ for CGCG 160095. All observations (June 1996 and July 1997) were performed with an elementary integration time of $5.04 \mathrm{~s}$ and a gain of 2. For all June 1996 observations the number of integrations for each raster position is $\simeq 10$. This number was doubled for the first raster position to allow for the detector stabilization. The July 1997 observations were obtained with $\simeq 20$ exposures per raster position, except for the first position $(\simeq 40)$.

The data reduction was performed with the CAM Interactive Analysis (CIA) ${ }^{1}$ software. For each raster position a flat field was obtained from the median of the remaining raster positions. To correct for glitches we used the multi-resolution method (Starck et al. 1999): events that change more rapidly than a given time scale and with fluxes higher (or lower) than $n$ times the rms are considered glitches and they are masked. To make sure that none of the sources are recognized as a glitch, we chose $n=5$ and $t$ such that $2^{t}$ is less than the number of readouts for each position in the sky. The main difficulty in the reduction of ISOCAM images is that of properly accounting for the time hysteresis of the detector. The initial pixel response to an incoming flux $\left(I_{\mathrm{i}}\right)$ is in fact such that $I\left(t_{0}\right)=f \cdot I_{\mathrm{i}}$ with $0<f<1$. The correct intensity $I_{\mathrm{i}}$ is reached only after some stabilization time, which varies from pixel to pixel and depends on the "history" of each pixel. The transient behavior is very difficult to model because it is non linear to the incoming radiation. To correct data for the transient effects we used the inversion model developed at the Institut d'Astrophysique Spatiale (Abergel et al. 1996; Starck et al. 1999). This method assumes that 1) the initial pixel response is $60 \%$ of the real flux and 2) the stabilization time decreases

1 CIA is a joint development by the ESA Astrophysics Division and the ISOCAM consortium let by ISOCAM PI, C. Cesarsky, Direction des Sciences de la Matière, C.E.A., France. 
as the incoming flux increases and it depends on the pixel previous history. As this method fails in the first read-outs for the lack of previous data, we have masked some first read-outs. The number of masked frames changes slightly from observation to observation, depending on how much time the stabilization takes (i.e. from the history of previous observations). Finally, only images taken in the LW2 filter were further corrected for the effect of a large spatial scale transient in order to obtain uniform background levels at each raster position. Assuming the background level of the last raster position correct, we normalize the background of the remaining raster positions to this level. We checked that this procedure produces more uniform background levels throughout the image without affecting the target flux. We remark that the ISOCAM data reduction is still preliminary. A better comprehension of the transient behavior of the detector will allow us to reduce the uncertainties associated with the present photometric measurements, which are estimated to be $\sim 30 \%$.

\subsection{ISOPHOT data}

Observations were made with the ISOPHOT (Lemke et al. 1996) instrument in the period 18 June 1996 to 2 July 1996, using the C100 and C200 detector arrays. Only data taken with the $2 \times 2$ pixel $\mathrm{C} 200$ detector array are reported here. As each galaxy could be contained within the $180^{\prime \prime} \times$ $180^{\prime \prime}$ field of view of the array, each was observed in a single spacecraft pointing. During the pointing the focal plane chopper was used to alternate between exposures of the centrally positioned target and exposures of a reference field offset by $-180^{\prime \prime}$ in the spacecraft $Y$ dimension. This placed the reference field between 198 and 210 degrees E of $\mathrm{N}$ for the encountered combinations of range of epoch and position. Data was taken using the filter sequence C200, C120 and finally C160 for each galaxy pointing. Rectangular approximations to the bandpasses of these filters are 170-238, 95-142 and 130-218 $\mu \mathrm{m}$, respectively. Typically, for each filter $16 \mathrm{~s}$ were spent on source and $16 \mathrm{~s}$ off source, split between 4 chopper cycles.

Version 7.3.2 of the ISOPHOT Interactive Analysis package PIA (Gabriel et al. 1997) was used to linearize the detector response, remove the dark current, and to convert the signal from engineering units into MJy/steradian. For the latter, standard (library) detector responsivities were used, as the detector never equilibrate in response to the illumination with the on-board calibration source. The derived brightness towards the reference position were found to be scattered with an rms of some $20 \%$ of the expected signal, which is predicted to be dominated by the smooth zodiacal light, rather than structured cirrus emission even at the wavelengths observed. This scatter is an indication of the absolute calibration accuracy of the observations. The systematic variation in detector responsivity during the sequential measurements in the filters should however be substantially smaller than that between observations of galaxies, resulting in a more accurate determination of galactic color.

To derive the source flux densities, account must be taken of signal losses due to detector hysteresis in the chop cycle, the presence of source emission in the reference position due to diffraction, and the effect of the socalled "vignetting" on the illumination of the detector array. Furthermore, due to the strong undersampling of the four pixels on the on source position, symmetrical about the galaxy with no pixel pointing directly at the galaxy, the derivation of integrated flux densities is strongly dependent on the beam response pattern assumed. The combined effect of these factors means that integrated flux densities derived from a simple-minded subtraction of the reference signals from the source signals followed by a summation of the on-source signals from the 4 detector pixels are too low by some $100 \%$.

The strategy adopted to derive the source flux was therefore to simulate the response of the detector to a point source on a flat background until the simulated signals matched the observed signals as a function of the point source flux density and background brightness. The assumption of a point source is reasonable when the FWHM beamsize of c.a. 100" is compared with the anticipated extent of the FIR emission of less than half of the extent to the 25 th $B$ magnitude (cf. Table 1 ). In the simulations beams in each filter were taken from the calibration data accompanying PIA. Correction factors for "vignetting" were derived from specific calibration measurements of this effect using the C160 filter (target identifier TDT $=1104203$ ) and assumed to hold for all three filters. In simulating the detector hysteresis the FouksSchubert model for the response of the detector to illumination steps (Schubert et al. 1995) was adopted, using a $\beta$ parameter of 0.85 and adapting the exponential time constant to the observed responsivity variation after switch on of the focal plane calibration sources.

Some verification of the flux densities derived from the overall simulation procedure is given by the fact that the photometry in the $C_{120}$ filter agrees within the errors with that seen in the spectrally overlapping IRAS 100 micron filter for those sources detected with IRAS, with a $100 / 120 \mu \mathrm{m}$ ratio of 1.0 . The total PHOT error on the density flux are estimated to be $\simeq 30 \%$. To check the consistency of the PHOT flux we plot in Fig. 1 the $F_{120}$ as function of $F_{100}$. The IRAS $100 \mu \mathrm{m}$ filter ranges from 65 to $145 \mu \mathrm{m}$ and the $C_{200}$ PHOT filter at $120 \mu \mathrm{m}$ from 95 to $142 \mu \mathrm{m}$. Thus, these two filters have significant overlap. The solid line in the figure represents the one-to-one correlation. The formal regression gives a slope of $0.87 \pm 0.1$. However the error budget, which is dominated by the ISOPHOT errors $(30 \%)$, allows us to conclude that the IRAS and ISOPHOT flux agree within the errors. 
Table 3. MIR (ISOCAM) and FIR (ISOPHOT) integrated flux

\begin{tabular}{|c|c|c|c|c|c|c|c|c|c|c|}
\hline CGCG & $\begin{array}{c}6.75 \mu \mathrm{m} \\
\mathrm{mJy} \\
(2)\end{array}$ & $\mathrm{rms}$ & $\begin{array}{r}15 \mu \mathrm{m} \\
\mathrm{mJy} \\
(4)\end{array}$ & $\mathrm{rms}$ & $\begin{array}{r}120 \mu \mathrm{m} \\
\mathrm{Jy} \\
(6)\end{array}$ & (7) & $\begin{array}{r}175 \mu \mathrm{m} \\
\mathrm{Jy} \\
(8)\end{array}$ & $\mathrm{rms}$ & $\begin{array}{r}200 \mu \mathrm{m} \\
\mathrm{Jy} \\
(10)\end{array}$ & $\mathrm{rms}$ \\
\hline 97062 & 8.16 & 2.45 & 7.26 & 2.18 & 0.50 & 0.20 & 0.58 & 0.05 & 0.45 & 0.12 \\
\hline 97073 & 5.45 & 1.63 & 6.82 & 2.04 & 0.90 & 0.18 & 0.63 & 0.06 & 0.45 & 0.16 \\
\hline 97079 & 7.12 & 2.13 & 8.33 & 2.50 & 0.72 & 0.14 & 0.48 & 0.15 & 0.54 & 0.35 \\
\hline 97087 & 38.84 & 11.65 & 43.45 & 13.03 & 2.95 & 0.31 & 3.12 & 0.31 & 2.25 & 0.36 \\
\hline 97138 & 3.47 & 1.00 & 2.39 & 0.71 & 0.63 & 0.07 & 0.44 & 0.06 & 0.31 & 0.41 \\
\hline 160020 & 13.64 & 4.10 & 15.11 & 4.53 & 0.58 & 0.24 & 0.87 & 0.08 & 0.89 & 0.31 \\
\hline 160026 & 9.77 & 2.93 & 10.64 & 3.20 & 0.81 & 0.39 & 0.69 & 0.07 & 0.34 & 0.27 \\
\hline 160055 & 29.48 & 8.84 & 34.63 & 10.38 & 2.65 & 0.34 & 2.42 & 0.37 & 2.31 & 0.35 \\
\hline 160067 & 12.52 & 3.75 & 14.00 & 4.20 & 0.66 & 0.11 & 0.60 & 0.05 & 0.20 & 0.24 \\
\hline 160076 & 2.73 & 0.82 & 2.33 & 0.70 & 0.38 & 0.10 & 0.57 & 0.14 & 0.36 & 0.20 \\
\hline 160086 & 6.66 & 2.00 & 6.33 & 1.90 & 0.38 & 0.12 & 0.33 & 0.06 & 0.60 & 0.60 \\
\hline 160088 & 23.33 & 7.00 & 22.36 & 6.70 & 0.92 & 0.16 & 1.03 & 0.12 & 0.84 & 0.15 \\
\hline 160095 & 21.73 & 6.51 & 19.50 & 5.85 & 0.85 & 0.19 & 1.16 & 0.12 & 0.94 & 0.37 \\
\hline 160127 & 4.31 & 1.29 & 4.19 & 1.26 & 0.51 & 0.13 & 0.41 & 0.08 & 0.28 & 0.25 \\
\hline 160128 & 4.27 & 1.28 & 4.87 & 1.46 & 0.50 & 0.25 & 0.57 & 0.09 & 0.59 & 0.30 \\
\hline 160139 & 4.28 & 1.28 & 6.06 & 1.83 & 0.49 & 0.13 & 0.43 & 0.05 & 0.18 & 0.12 \\
\hline 160252 & 28.30 & 8.50 & 34.05 & 10.20 & 2.59 & 0.43 & 2.37 & 0.33 & 2.06 & 0.76 \\
\hline 160260 & 50.34 & 15.10 & 54.39 & 16.32 & 2.43 & 0.44 & 1.96 & 0.25 & 1.45 & 0.47 \\
\hline
\end{tabular}

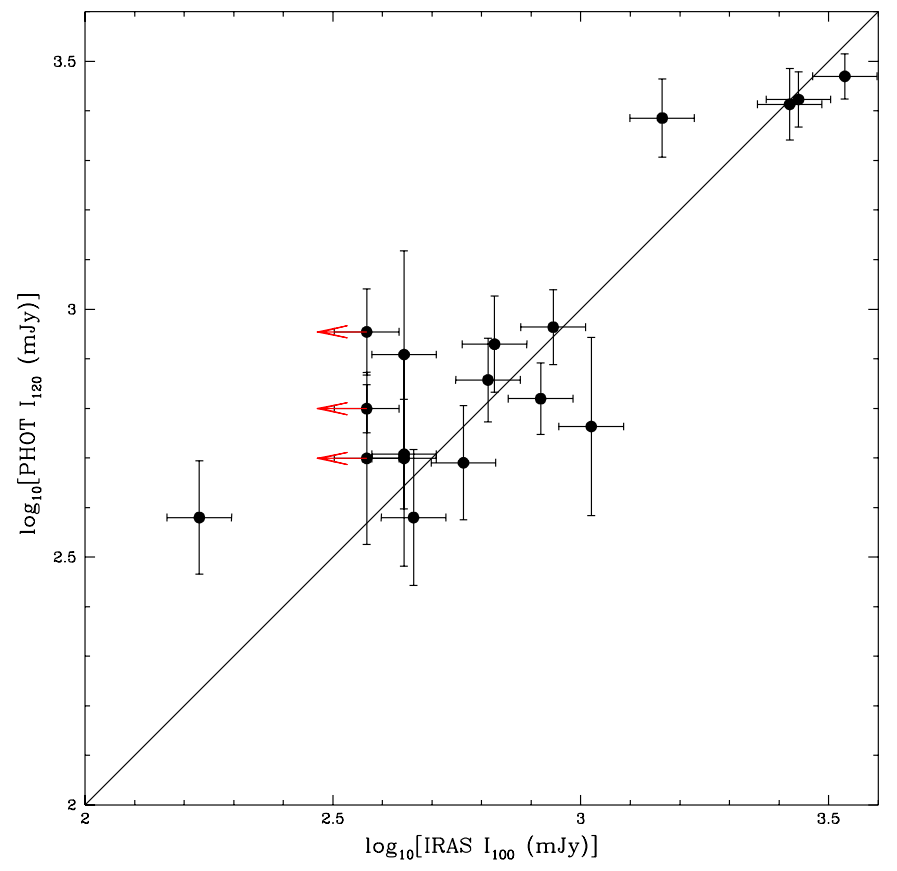

Fig. 1. The 120 (PHOT) flux as function of the 100 (IRAS) flux for all the observed galaxies. The solid line represents the one-to-one correlation

\section{Results}

\subsection{MIR and FIR flux}

All galaxies but one have been detected at all wavelengths. Twelve out of eighteen galaxies are not resolved by ISOCAM. MIR images of the resolved galaxies (CGCG 160055, CGCG 160088, CGCG 160095, CGCG 160252, CGCG 160260 and CGCG 97087) at 6.75 and $15 \mu \mathrm{m}$ are shown in Fig. 2. Observations taken in July 1996 and June 1997 have been averaged after rebinning to the same pixel size (i.e. $3^{\prime \prime} /$ pixel only for CGCG 160055 and CGCG 160252). Integrated flux in the MIR and FIR bands of all target galaxies are given in Table 3 organized as follows:

Column 1: CGCG name;

Columns 2, 3: $6.75 \mu \mathrm{m}$ flux with uncertainty (in mJy);

Columns 4, 5: $15 \mu \mathrm{m}$ flux with uncertainty (in mJy);

Columns 6, 7: $120 \mu \mathrm{m}$ flux with uncertainty (in Jy);

Columns 8, 9: $175 \mu \mathrm{m}$ flux with uncertainty (in Jy);

Columns 10, 11: $200 \mu \mathrm{m}$ flux with uncertainty (in Jy).

The background levels at 7 and $15 \mu \mathrm{m}\left(\mathrm{mJy} / \operatorname{arcsec}^{2}\right)$ are listed in Table 4 and are consistent with those extrapolated from the IRAS all-sky survey.

\subsection{Positional uncertainty of the ISOCAM observations}

The celestial coordinates of the target galaxies are known with $\sim 1^{\prime \prime}$ accuracy (see Gavazzi \& Boselli 1996). The LW2 and LW3 positions are consistent with one another because they were obtained in rapid sequence during the same satellite orbit and they are expected to be known with $6^{\prime \prime}-10^{\prime \prime}$ uncertainty (ISO Observer's Manual). Actually, the absolute pointing accuracy of the ISO satellite is $\sim 3^{\prime \prime}$, but most of the position uncertainties comes 
A. Contursi et al.: MIR and FIR properties of late-type cluster galaxies: ISO observations

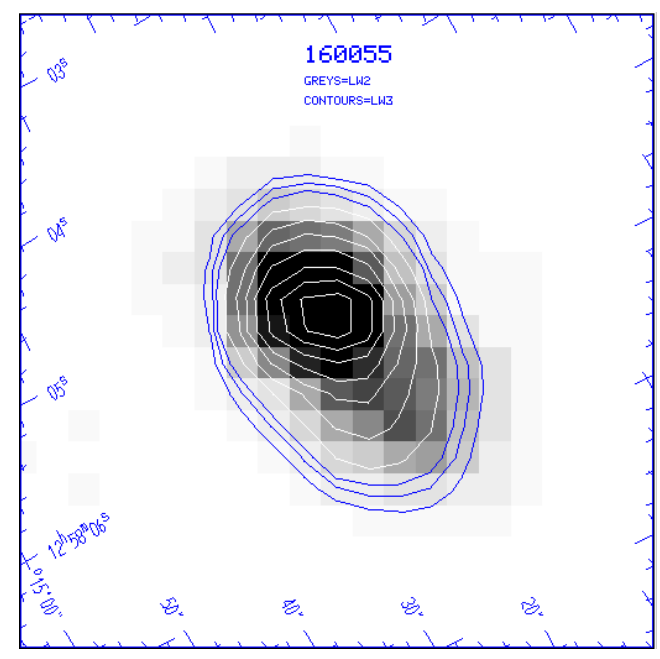

(a)

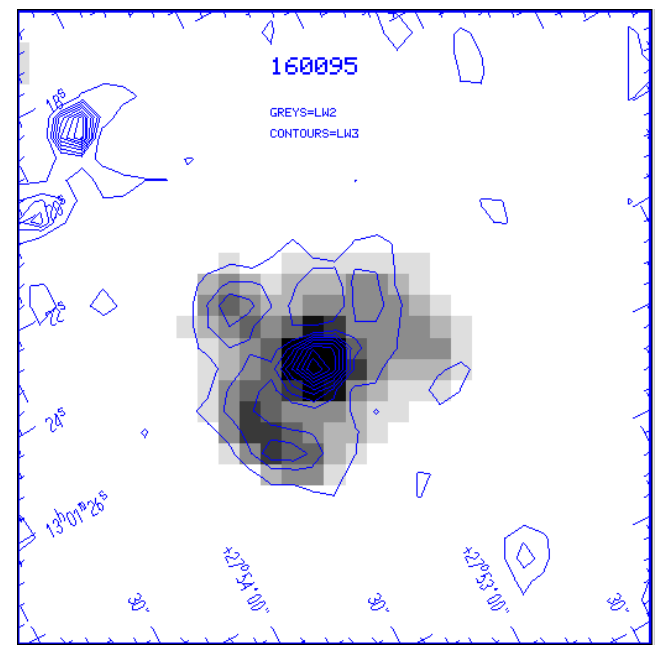

(c)

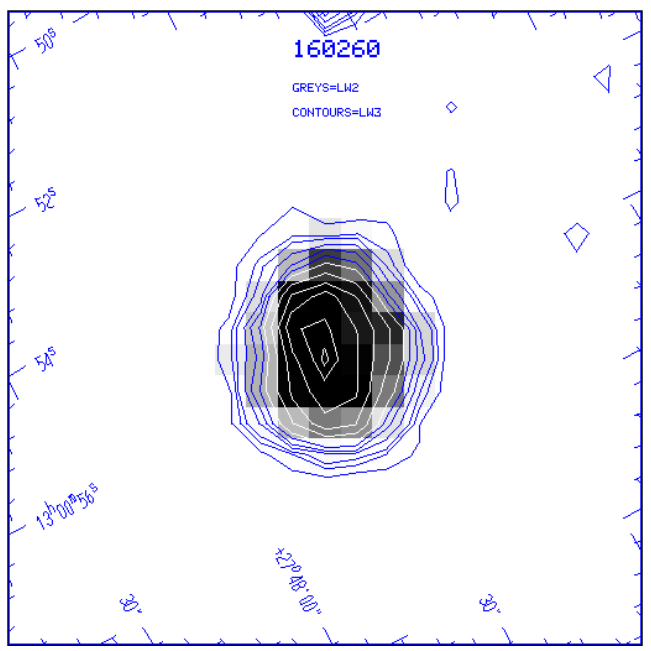

(e)

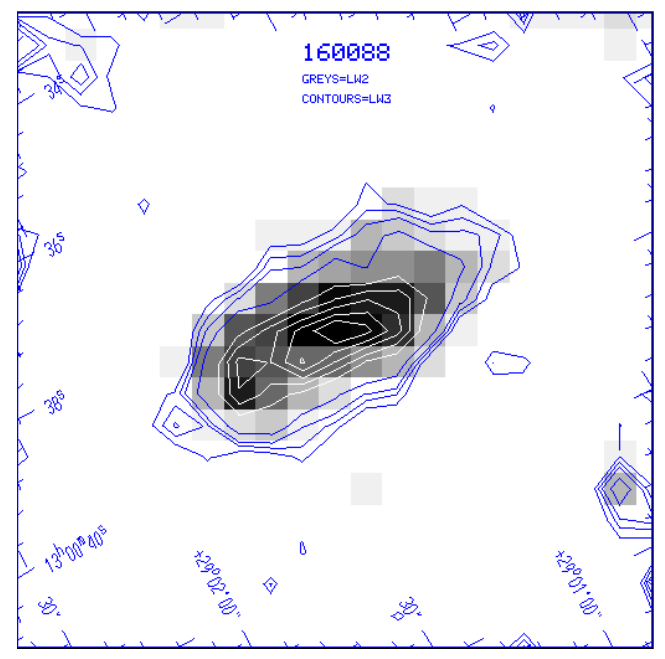

(b)

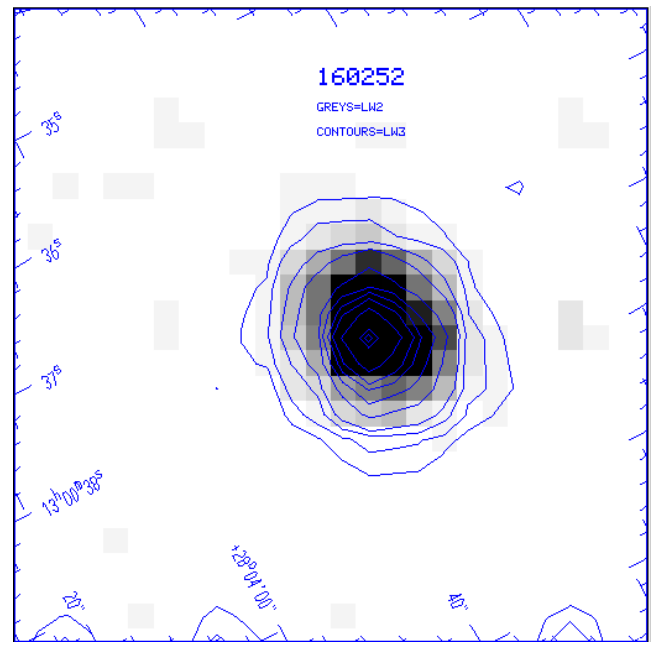

(d)

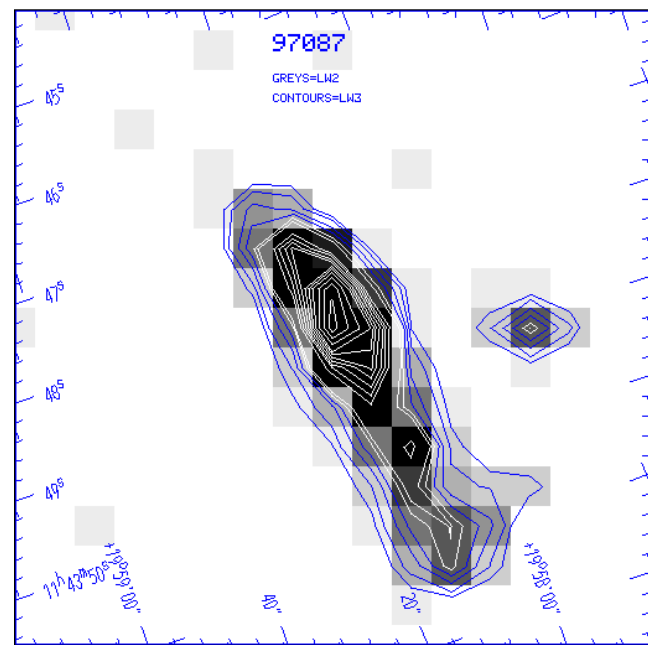

(f)

Fig. 2. ISOCAM images of galaxies CGCG 160055, CGCG 160088, CGCG 160095, CGCG 160252, CGCG 160260 and CGCG 97087. Contours at $15 \mu \mathrm{m}$ (LW3) are superposed to the $6.75 \mu \mathrm{m}$ (LW2) maps 
Table 4. The background average levels in the LW2 and LW3 filters for each cluster

\begin{tabular}{llll}
\hline & $6.75 \mu \mathrm{m}$ & $15 \mu \mathrm{m}$ \\
& & $\mathrm{mJy} / \operatorname{arcsec}^{2}$ & \\
\hline Coma & 0.09 & & 0.48 \\
A1367 & 0.16 & & 0.84 \\
\hline
\end{tabular}

from the jitter lens displacement, giving a total error up to $\sim 10^{\prime \prime}$. The average offsets between the optical and MIR peaks (assumed to be coincident) of the present data are found to be $3.5^{\prime \prime}$ and $5.7^{\prime \prime}$ in RA and Dec respectively. Some objects have individual positional errors up to $10^{\prime \prime}$. Therefore MIR images have been re-centered on the optical ones.

\subsection{MIR surface brightness distribution and star formation properties}

Most of the observed galaxies are faint and/or not resolved by CAM. It is thus not possible to analyze their MIR surface brightness distribution in any detail. Images in the LW2 filter are marginally more extended than the LW3 ones. This is partly an artifact due to the higher background at $15 \mu \mathrm{m}$ than at $6.75 \mu \mathrm{m}$ (see Table 4 ). The LW2 and LW3 surface brightness distribution do not significantly differ. The MIR emission is generally peaked at the center and decreases smoothly throughout the disk. However CGCG 97087, CGCG 160088 and CGCG 160095 show an extended IR emission and some structure. Some secondary peaks are visible at $6.75 \mu \mathrm{m}$ and not at $15 \mu \mathrm{m}$ probably because of the slightly different PSF at the two wavelengths.

Figures 3 and 4 show the comparison between the $6.75 \mu \mathrm{m}$ (LW2) and the $\mathrm{H} \alpha$ surface brightness distributions smoothed at the ISO resolution $\left(\simeq 7^{\prime \prime}\right)$ for the resolved galaxies ${ }^{2}$. $\mathrm{H} \alpha$ images taken from Gavazzi et al. (1998), refer to the "net" frames (obtained by subtracting the off-band from the on-band frames) and are taken as indicators of the galaxies star formation rates (SFRs). The majority of the resolved galaxies have their MIR emission more extended than the net $\mathrm{H} \alpha$ emission. In CGCG 97138 (not shown here), CGCG 160088 and CGCG 160260 (see Figs. 2 and 3 ) the MIR emission is centrally peaked, contrary to the $\mathrm{H} \alpha$ distribution.

The analysis of the spectral energy distribution of a complete, optically selected sample of late-type galaxies in the Virgo cluster has shown that their MIR emission could arise from both dust or photospheric emission from cool stars (i.e. giants and supergiants), this last contribution being more important in quiescent, early-type spiral (Boselli et al. 1998). The total MIR emission of the target

\footnotetext{
2 The H $\alpha$ image of galaxy CGCG 160095 has been smoothed to one half of the ISO resolution. At a resolution of $7^{\prime \prime}$ the HII regions disappear, preventing us from studying the relationship between the MIR and the $\mathrm{H} \alpha$ morphology of this galaxy.
}

galaxies is mostly due to dust, because the 6.75 and $15 \mu \mathrm{m}$ flux are much higher than the Rayleigh-Jeans tail emission of the coolest stellar populations (see the Spectral Energy Distributions in Fig. 6). On the other hand, the fact that the MIR emission does always peak in the galaxy centers could suggest that here the stellar contribution is significant. To check this, we evaluated the cool stellar contribution at $6.75 \mu \mathrm{m}$ in the disks and the bulges of the resolved galaxies. We represent the coldest stellar population emission with a black body at $3500 \mathrm{~K}$, whose amplitude is chosen in order to fit the $J, H$ and $K$ flux. For each pixel in the $H$ images, we calculate the values that it would have at $6.75 \mu \mathrm{m}$ (LW2 filter) according to this black body emission. In other words we obtained the map of the stellar contribution at $6.75 \mu \mathrm{m}$. We finally performed photometry on these images smoothed at the ISO resolution, considering two apertures corresponding to the disks and the bulges, to evaluate the contribution of the stellar emission at $6.75 \mu \mathrm{m}$ and we compared it with the ISOCAM LW2 value. We find that the dust emission dominates at $6.75 \mu \mathrm{m}$ in both disks and bulges of all galaxies except in the bulge of CGCG 160095, where the stars contribute up to $50 \%$ of the total flux.

\subsection{CGCG 97087 (U6697)}

The MIR emission from this galaxy shows a well resolved extended structure allowing a more detailed analysis. This galaxy, extensively studied by Gavazzi et al. (1984, 1995b), shows a moderately blue color $((B-H)=2.2)$, and a strong star formation rate $(\mathrm{H} \alpha E . W .=74 \AA)$. Its headtail like radio continuum morphology and asymmetric HI profile were interpreted by Gavazzi et al. (1995b) as due to dynamical interaction with the intracluster medium (ICM). Figure $4 \mathrm{~b}$ shows the $6.75 \mu \mathrm{m}$ contours superimposed on the "net" H $\alpha$ map. Various HII regions, labeled following Gavazzi et al. (1984) form a ring-like structure. The $\mathrm{H} \alpha$ and MIR emission do not show a one-to-one correspondence. Although the $\mathrm{H} \alpha$ and MIR peaks generally coincide in position, the relative intensities do not match.

Figure 5 shows a one--dimensional scan along the major axis of CGCG 97087 in the $\mathrm{H}$ band, "net" $\mathrm{H} \alpha$ line, radio continuum at $1.4 \mathrm{GHz}$ and MIR. These profiles have been obtained by integrating the corresponding images (once smoothed to the MIR resolution) along the minor axis, normalized to A4, i.e. the region of maximum brightness, coinciding with the nuclear region of the galaxy (Gavazzi et al. 1984). The positions of the $\mathrm{H} \alpha, \mathrm{MIR}$ and radiocontinuum peaks are coincident. Remarkably, the MIR relative intensities of HII regions better match those in the radio continuum, than those of the $\mathrm{H} \alpha$.

\subsection{The spectral energy distributions}

The spectral energy distribution from the UV (2000 ̊) to $200 \mu \mathrm{m}$ of all the target galaxies are shown in Fig. 6 . 


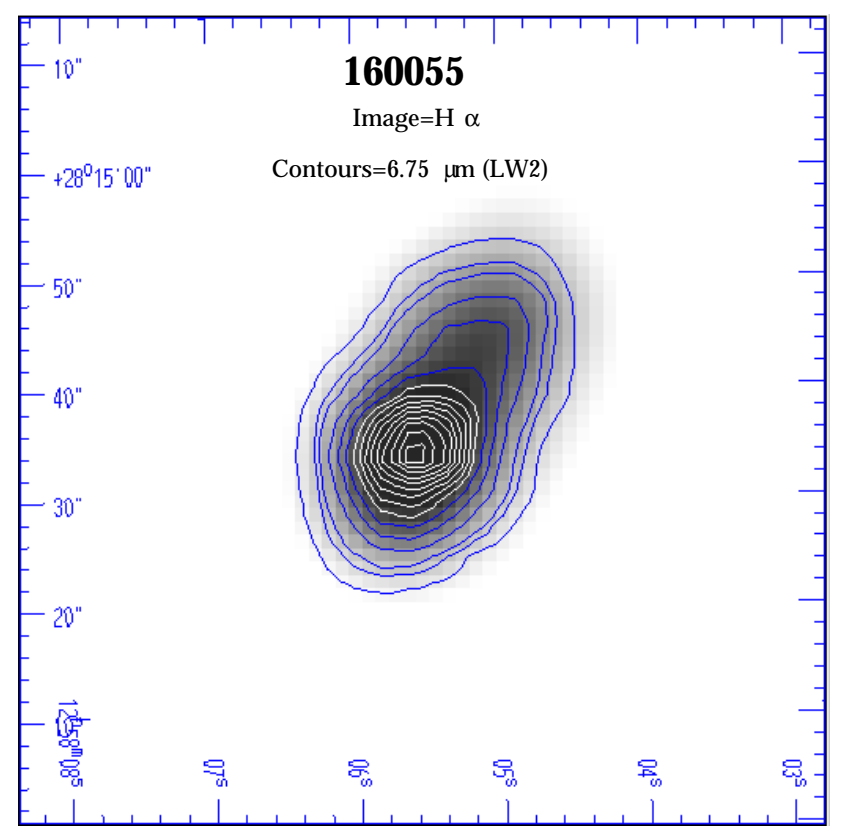

(a)

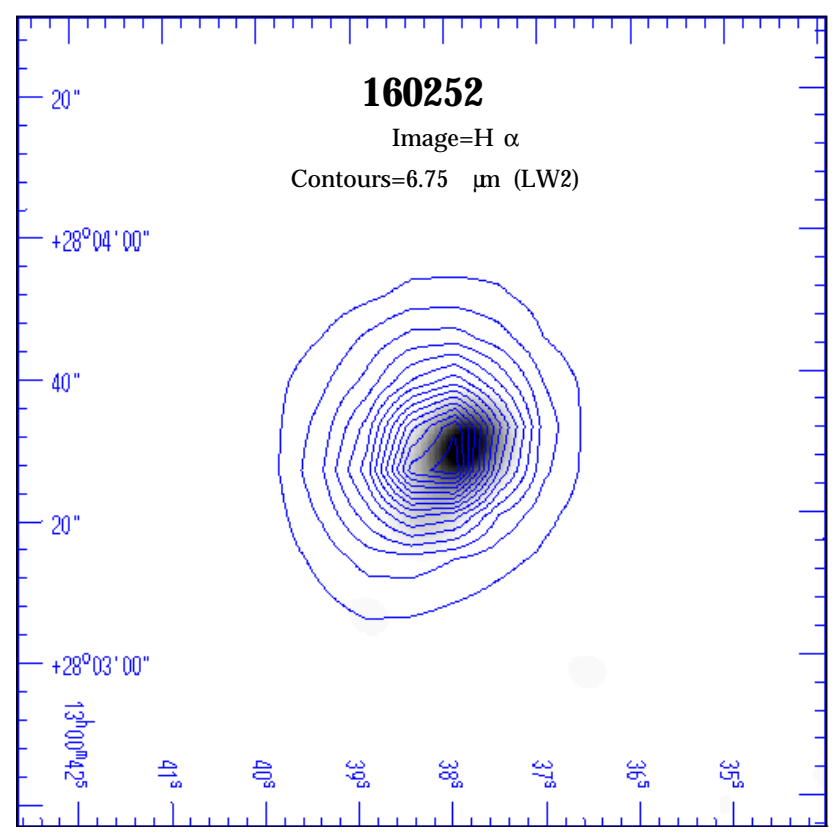

(c)

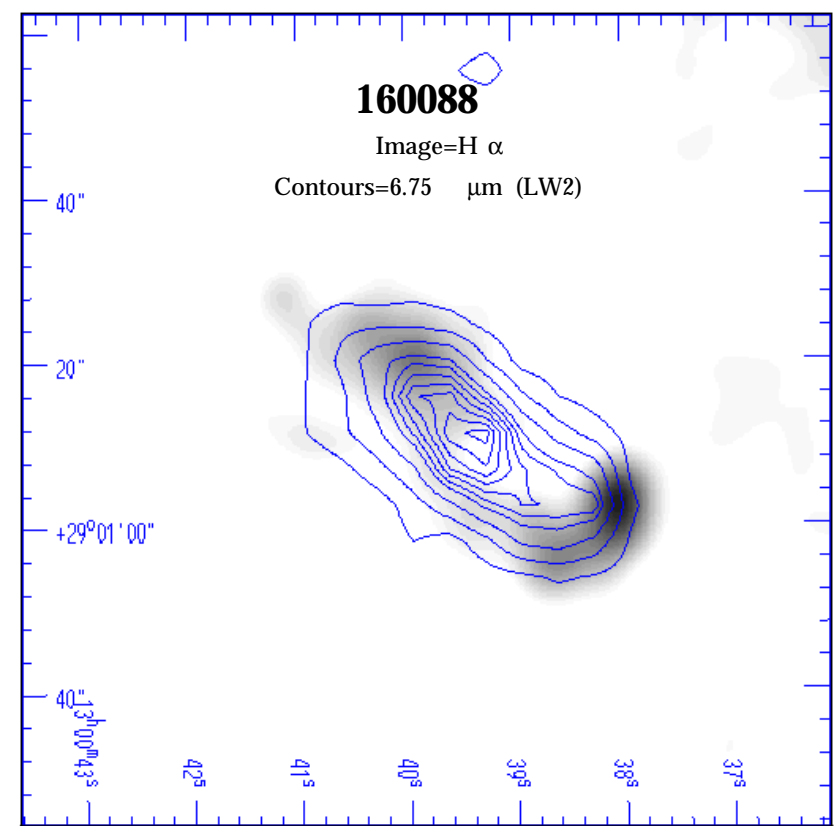

(b)

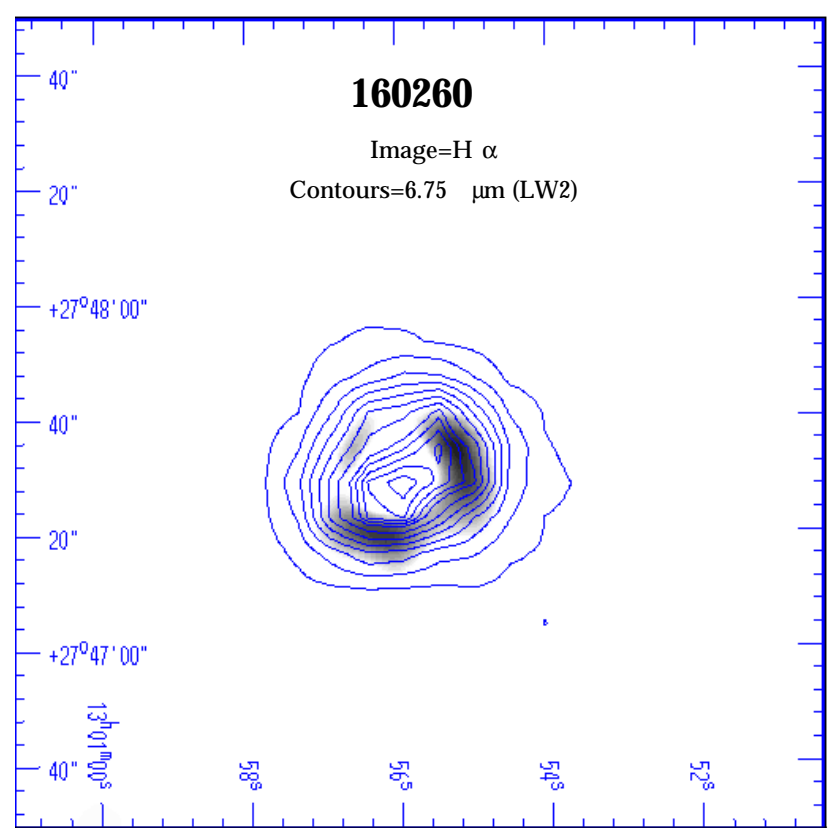

(d)

Fig. 3. $6.75 \mu \mathrm{m}$ (LW2) contours superposed to the $\mathrm{H} \alpha$ images (smoothed to the same resolution) of the resolved galaxies CGCG 160055, CGCG 160088, CGCG 160252 and CGCG 160260

Fluxes are normalized to the $H$ band flux which is a direct indicator of the galaxy total mass (Gavazzi et al. 1996). This allows us to remove the first-order luminositymass dependence. A black body curve at $3500 \mathrm{~K}$ has been used to represent the emission of the colder and older postsequence stars, which in principle may significantly contribute also at MIR wavelengths. For this component, we match the black body intensity to the NIR broad band photometry.
To represent the FIR data $(100,120175$ and $200 \mu \mathrm{m})$ we adopt a modified black body law $\left(B_{\nu} \cdot \nu^{2}\right)$ to take into account the emissivity of the big grains. This law is fitted to the data using a $\chi^{2}$ fit with two parameters: the temperature and the intensity (i.e. the solid angle $\Omega$, see Sect. 4.6). We cannot fit any component in the range between 20 and $60 \mu \mathrm{m}$ since we have only one detection and one upper limit at 60 and $25 \mu \mathrm{m}$ respectively. We do not fit the MIR (LW2 and LW3) data with any thermal 


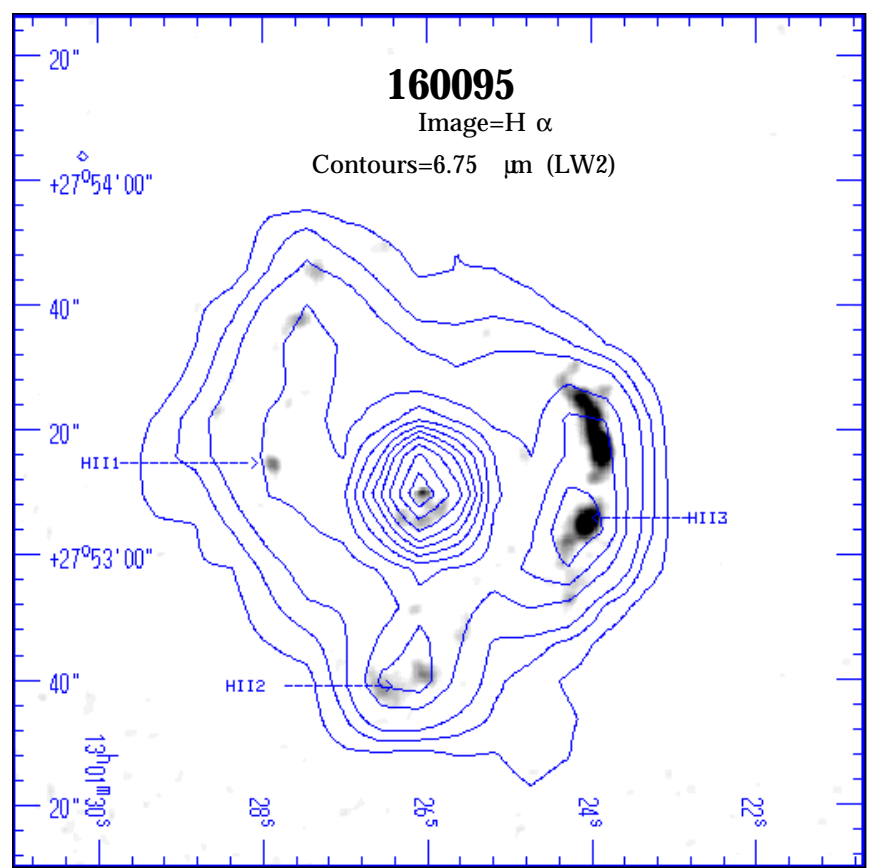

(a)

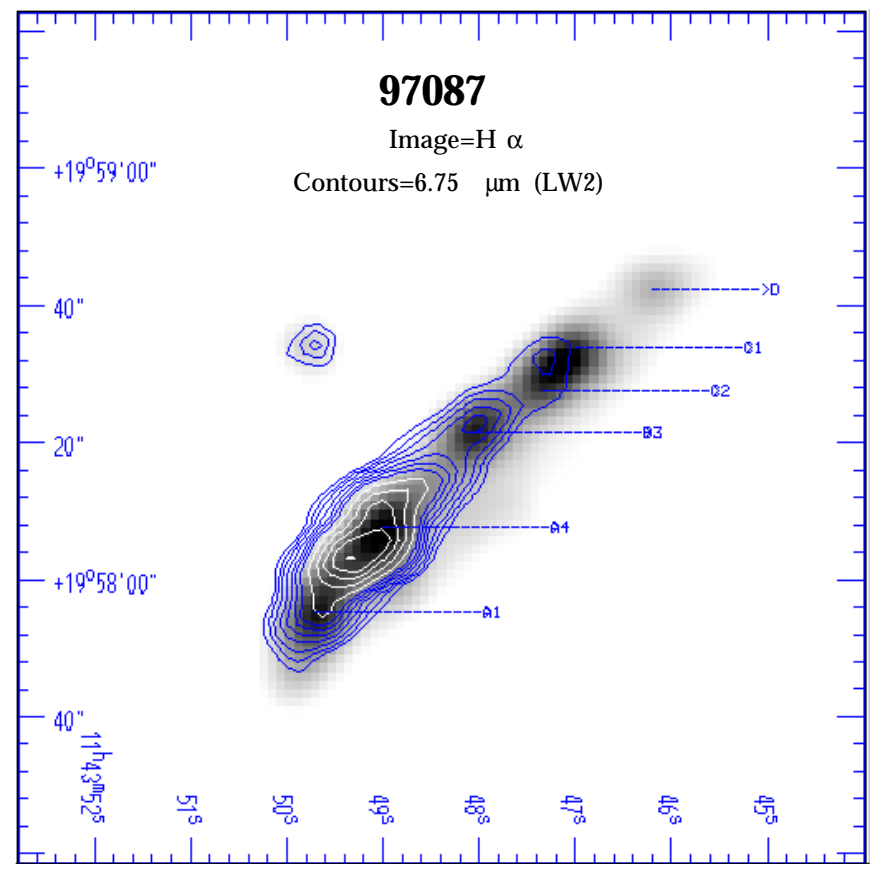

(b)

Fig. 4. Same as the Fig. 2 but for the best resolved galaxies CGCG 160095 and CGCG 97087. Labels mark the HII regions discussed individually in the text

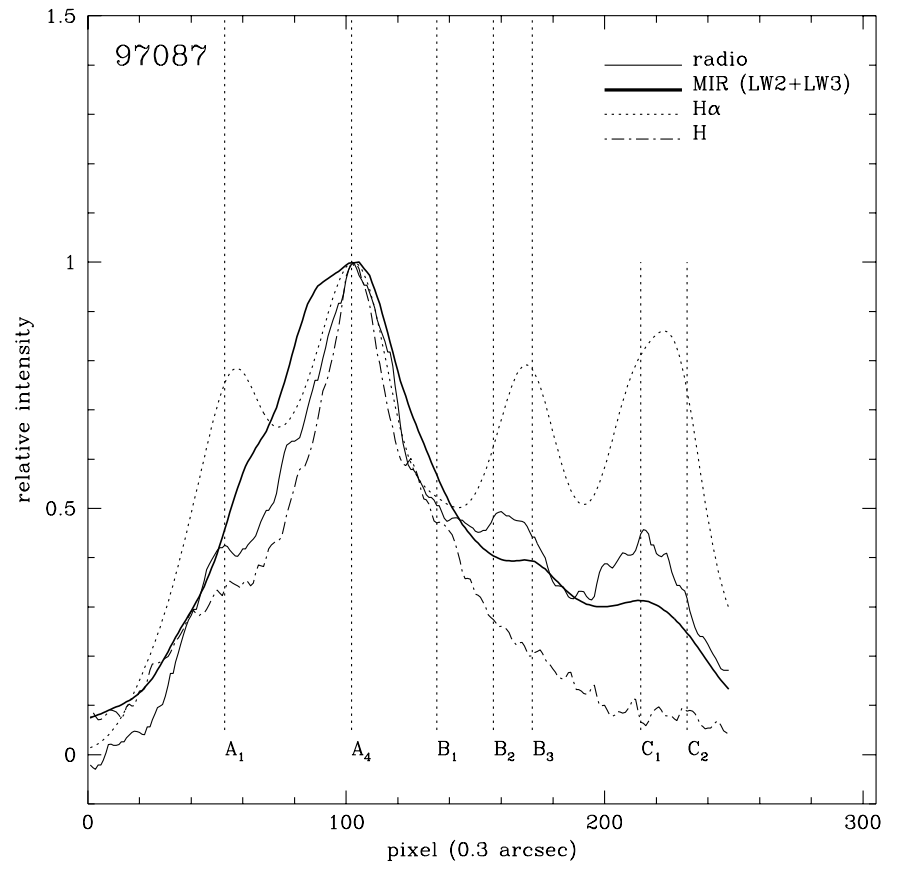

Fig. 5. One-dimensional profiles at various wavelengths along the plane of the galaxy CGCG 97087. Data have been smoothed to the ISOCAM resolution. Flux are normalized to the flux in $\mathrm{A} 4$

component, because these grains are not supposed to be in thermal equilibrium. The FIR fitted temperatures range from 17 to $29 \mathrm{~K}$ and they are listed in Col. 2 of Table 5 . We note that most of the galaxies have average temperatures between 20 and $23 \mathrm{~K}$. Temperature uncertainties derive from the uncertainty on the PHOT photometry, which is $\sim 30 \%$ and the uncertainty on the assumed big grain emissivity. To evaluate the latter uncertainty we fitted a modified black body from 100 to $200 \mu \mathrm{m}$ assuming an $\beta=1.5$. Grain emissivities larger than 2 or smaller than 1.5 have not been observed at these wavelengths in average interstellar medium conditions of normal galaxies (see Alton et al. 2000 and references therein). The temperatures obtained are $\sim 15 \%$ smaller than the values given in Table 5. Thus the total error on the fitted temperatures is $35 \%$.

\subsection{The dust content}

The SED fit parameters (temperature and intensity) can be used to derive the dust mass of the target galaxies via the relation:

$M_{\mathrm{d}}=\left(S_{\nu} \cdot D^{2}\right) /\left(K_{\nu} \cdot B_{\nu} \nu^{2}\right)$

where $S_{\nu}$ is the observed flux at the frequency $\nu ; D$ is the galaxy distance, $K_{\nu}$ is the mass absorption coefficient of grains, and $B_{\nu} \nu^{2}$ is the modified Planck law used to fit the FIR data. We adopt a Draine \& Lee (1984) model for grains. An exponent $\beta=2$ for the grain emissivity value seems the most appropriate for normal, late-type galaxies as determined from the spectral energy distribution of nearby galaxies observed in the FIR and submillimetric wavelength range (Bianchi et al. 1999b). The extrapolated mass absorption coefficient at $100 \mu \mathrm{m}$ for a $\nu^{2}$ emissivity law is equal to $K_{100 \mu \mathrm{m}}=90 \mathrm{~cm}^{2} \mathrm{~g}^{-1}$. Since $S_{\nu}=\Omega \cdot B_{\nu} \nu^{2}$, where $\Omega$ is the solid angle, $\log S_{\nu}=\log \left(B_{\nu} \nu^{2}\right)+\log \Omega$, the 

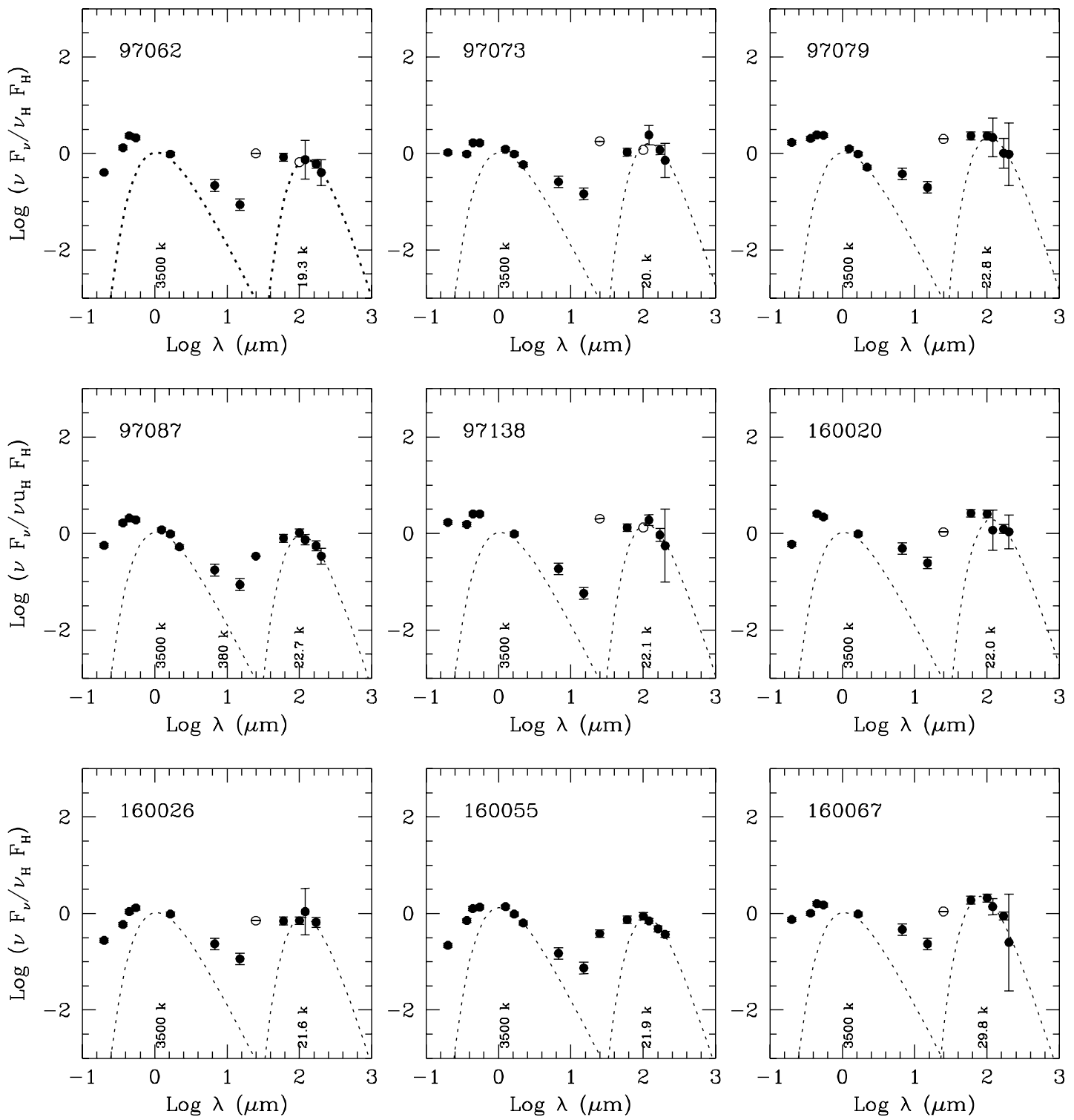

Fig. 6. Spectral Energy Distribution (SEDs) from the UV $(2000 \AA)$ to the FIR $(200 \mu \mathrm{m})$ of the sampled galaxies. Energies are normalized to the flux in the $H$ band $(1.65 \mu \mathrm{m})$. A black body at $T=3500 \mathrm{~K}$ has been used to represent the cold stellar population which may contribute also at MIR wavelengths. A gray body law has been fitted to the FIR 100-200 $\mu$ m data points. The dust fitted temperature are marked. Open circles indicate upper limits

last term being the offset necessary to fit the adopted law to the data. The derived dust masses and dust to gas ratios (see Sect. 5.4) are listed in Table 5.

\section{Discussion}

\subsection{AlB carriers}

The spectral energy distributions in Fig. 6 show that the MIR $(6.75$ and $15 \mu \mathrm{m})$ flux of all galaxies except CGCG 160095 are higher than the Rayleigh-Jeans tail of the coldest stellar component. This means that the total MIR emission in these galaxies is dominated by dust. The ISOCAM images of the galaxies resolved at 6.75 and $15 \mu \mathrm{m}$ clearly show that the dust component emitting at these wavelengths is rather uniformly distributed over the disks. Since MIR emission arises principally from aromatic macro-molecules and very small grains transiently heated primarily by UV photons we expect to detect MIR emission around the star forming regions. We instead observe MIR emission also away from the HII regions. For example, galaxies CGCG 160260 and CGCG 160095 (see Figs. 3 and 4) show a diffuse MIR emission throughout their disks also where no $\mathrm{H} \alpha$ emission is detected. We conclude that AIB carriers are present all over the disks and that, far from HII regions, they are excited by non-ionizing photons. This result is in agreement with other MIR observations. The Circular Variable Filter (CVF) data of the vdB133 reflection nebula presented in Uchida et al. (1998) and the CAM observations of M 31 (Pagani et al. 1999) clearly show that AIB carriers can 

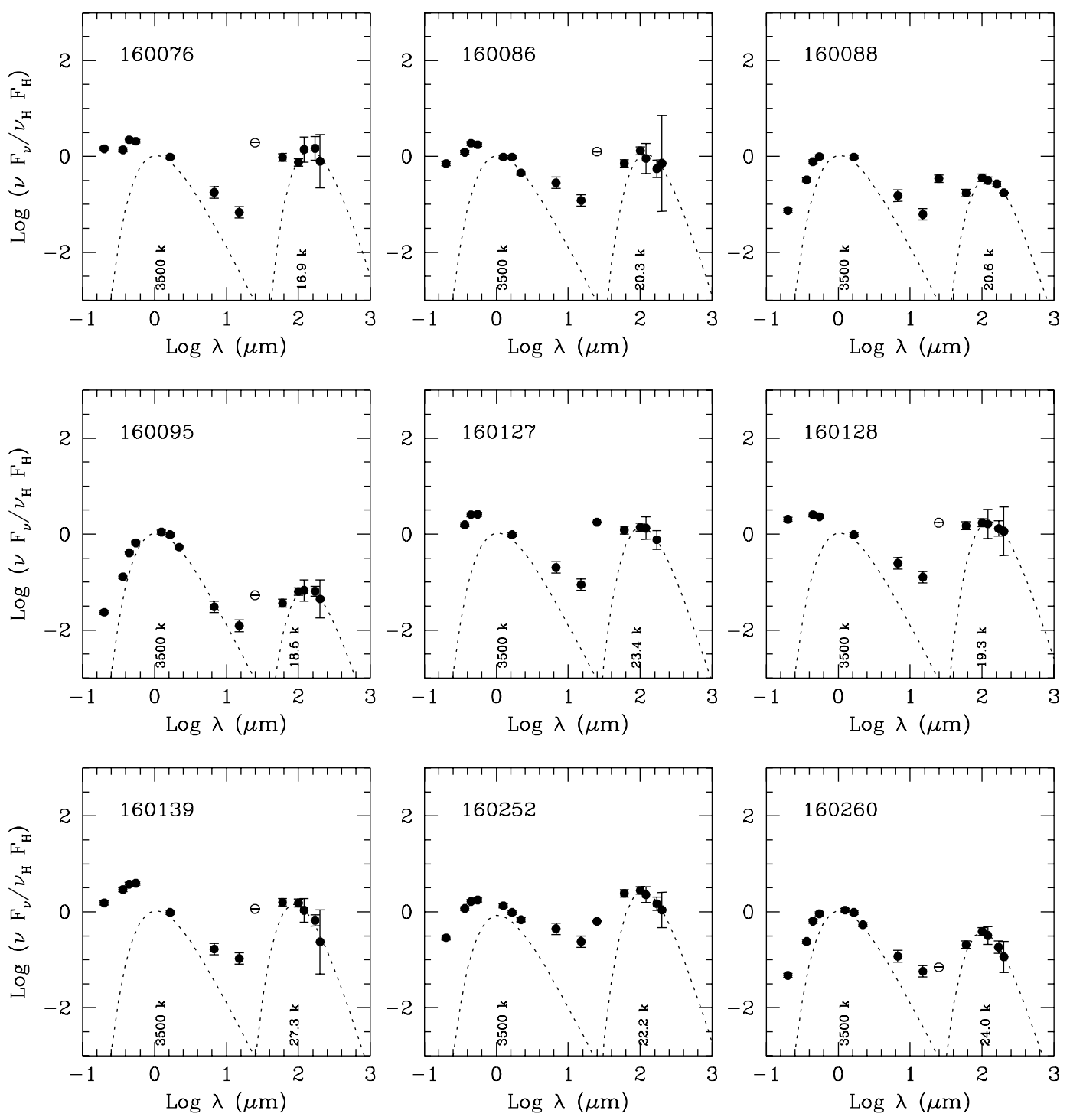

Fig. 6. Continued

be excited also by visible photons. In particular, Pagani et al. (1999) show that UV excitation dominates over visible excitation only when the $\mathrm{UV} /$ visible intensity ratio is larger than about 2 times that in the solar neighborhood. Indeed, one expects the same result in low metallicity environments where UV photons can freely travel far away from the sites of massive star formation, producing excitation of the AIB carriers all over the disks. However, this is unlikely for the analysed galaxies whose metallicities are in the range $0.7 Z_{\odot}<Z<1.5 Z_{\odot}$ (Gavazzi et al., in preparation).

The relationship between the MIR emission and the star formation properties of the galaxies under study is rather complex. The two reddest and most $\mathrm{HI}-$ deficient galaxies in the sample (CGCG 160260 and CGCG 160095) behave differently. CGCG 160260 hardly shows any MIR-$\mathrm{H} \alpha$ morphological correspondence, which could partly derive from the insufficient angular resolution of ISOCAM, while CGCG 160095, beside its diffuse MIR components, shows MIR emission peaks on the HII regions visible in
$\mathrm{H} \alpha$. The only other galaxy sample which shows MIR peaks on the HII regions is CGCG 97087. However, this galaxy has star formation properties very different from those of CGCG 160095, the former galaxy being one of the bluest and most active sources, the latter being the reddest and most quiescent galaxy in our sample. In Sect. 4.4 we remarked that the relative intensities of the $\mathrm{H} \alpha$ features in CGCG 97087 (Fig. 5) do not match those in the MIR. The ISRF in these regions could be sufficiently high to destroy the AIB carriers (Boulanger et al. 1988) or to heat VSGs to temperatures such that their bulk emission falls in the LW3 wavelength range. Boselli et al. (1997a) found that in late-type Virgo and Coma galaxies the global MIR flux increases with the UV flux only at low and intermediate star formation rates. At higher UV flux the $7 \mu \mathrm{m}$ and, to a lesser extent, the $15 \mu \mathrm{m}$ flux densities, cease to increase with increasing SFR probably because of AIBs destruction. CGCG 97087 is a typical object in this regime.

Cesarsky et al. (1996) and Contursi et al. (1998, 2000) showed that for UV ISRF higher than $10^{3}$ times that 
Table 5. The cold dust temperatures, apparent dust masses and apparent total dust to gas $\left(M_{\mathrm{HI}}+M_{\mathrm{H}_{2}}\right)$ ratios. For $\mathrm{CO}$ undetected galaxies the total dust to gas ratio is an upper limit. For 160076 , not observed in CO, we assume $M_{\mathrm{H}_{2}}=0.1 \times M_{\mathrm{HI}}$ as in Boselli et al. (1997b)

\begin{tabular}{crrrr}
\hline CGCG & $T_{\text {dust }}$ & $M_{\text {dust }}$ & \pm & $M_{\text {dust }} / M_{\text {gas }}$ \\
& $\mathrm{K}$ & $10^{6} M_{\odot}$ & $10^{6} M_{\odot}$ & $10^{-3}$ \\
$(1)$ & $(2)$ & & $(3)$ & $(4)$ \\
\hline 97062 & 19.3 & 3.5 & 1.2 & 2.8 \\
97073 & 20.0 & 3.3 & 1.1 & 6.6 \\
97079 & 22.8 & 1.9 & 0.7 & 1.7 \\
97087 & 22.7 & 9.8 & 3.4 & 4.8 \\
97138 & 22.1 & 1.6 & 0.5 & 2.1 \\
160020 & 20.8 & 4.7 & 1.6 & 6.9 \\
160026 & 21.6 & 2.5 & 0.9 & 6.1 \\
160055 & 21.9 & 11.4 & 4.0 & 4.4 \\
160067 & 29.8 & 0.6 & 0.2 & 1.1 \\
160076 & 17.0 & 6.3 & 2.2 & 9.4 \\
160086 & 20.3 & 2.8 & 1.0 & 8.2 \\
160088 & 20.6 & 5.4 & 1.9 & 2.3 \\
160095 & 18.5 & 9.4 & 3.3 & 3.2 \\
160127 & 23.4 & 1.3 & 0.5 & 1.5 \\
160128 & 19.3 & 4.4 & 1.5 & 6.1 \\
160139 & 27.4 & 0.6 & 0.2 & 0.4 \\
160252 & 22.2 & 10.0 & 3.5 & 5.6 \\
160260 & 24.0 & 5.9 & 2.0 & 1.7 \\
\hline
\end{tabular}

of the solar neighborhood the $15 / 6.75 \mu \mathrm{m}$ ratio increases. This might be due either to the destructions of AIB carriers that dominate the emission at $6.75 \mu \mathrm{m}$ or to an increase of the $15 \mu \mathrm{m}$ emission due to the contribution of VSGs heated to higher temperatures. To check this hypothesis, we measured the MIR flux of the resolved HII regions marked in Figs. 4a and 4b of CGCG 160095 and CGCG 97087. The $15 / 6.75 \mu \mathrm{m}$ ratios are listed in Table 6 .

The fact that the $15 / 6.75 \mu \mathrm{m}$ ratios of HII regions in CGCG 97087 are higher than those in CGCG 160095 shows that destruction of AIB carriers and/or the heating of VSGs or BGs at high temperatures are more likely going on in CGCG 97087. In CGCG 160095, where the stellar contribution to the MIR emission is important (see Fig. 7), the interstellar radiation field is probably not sufficient to destroy AIBs carriers, even in its HII regions.

\subsection{Very small grains}

The average $15 / 6.75 \mu \mathrm{m}$ flux ratio of the present galaxies is $\simeq 1$, very similar to the value found by Cesarsky et al. (1996a) and by Abergel et al. (1996) for relatively quiet regions in our Galaxy such as NGC 7023 and $\rho$ Ophiuchi and by Pagani et al. (1999) in M 31. These galaxies lie also in the quiescent region of the Vigroux et al. (1999) and Dale et al. (2000) IR color-color diagram, which plots the $6.75 / 15 \mu \mathrm{m}$ CAM ratio as a function of the $60 / 100 \mu \mathrm{m}$ IRAS colors, the latter ranging from 0.3 to 0.7 . Their MIR colors are also similar to those of normal spiral galaxies belonging to the Virgo sample analyzed by Boselli et al. (1998). Values of $15 / 6.75 \mu \mathrm{m}>1$ are expected in regions where the UV ISRF becomes higher than $10^{3-4}$ times the local ISRF (Cesarsky et al. 1996b, 1996a; Contursi et al. 1998) and lower values where the contribution of the cold stellar emission at $6.75 \mu \mathrm{m}$ becomes relevant. The increasing of the $15 / 6.75 \mu \mathrm{m}$ with the galaxy activities is due to the increasing contribution of hot VSGs at $15 \mu \mathrm{m}$ and the partial destruction of AIB carriers in high interstellar radiation field environments. Thus, for the observed galaxies, the global contribution of VSG or hot BG emission in the wavelength range covered by the LW3 filter is small or negligible. The average ISRF in the target galaxies is never high enough to heat VSGs to high temperatures $(\simeq 300 \mathrm{~K})$ except in highly excited HII regions. Thus, the bulk of the emission at MIR wavelengths is primarily due to the AIB carriers. These MIR properties are characteristic of the photo-dissociation regions (PDRs) (Cesarsky et al. 1996b; Contursi et al. 1998, 2000).

In normal galaxies we expect the bulk of the VSG emission being at $\lambda>20 \mu \mathrm{m}$. Unfortunately most galaxies in our sample are undetected by IRAS at $25 \mu \mathrm{m}$. However, the SEDs of the four galaxies detected at $25 \mu \mathrm{m}$ (see Fig. 6) clearly show that the fitted FIR cold component between 100 and $200 \mu \mathrm{m}$ cannot account for the 25 and $60 \mu \mathrm{m}$ observed flux, so that another hotter component has to be invoked. Our sample as a whole provides further evidence for such a component: the $60 \mu \mathrm{m}$ data point often lies above the FIR fitted component and the upper limits at $25 \mu \mathrm{m}$ do not rule out its existence. This additional dust component could arise from both VSGs and/or BGs hotter than those contributing at $\lambda>100 \mu \mathrm{m}$. Consequently our data are not in disagreement with the Désert et al. (1990) dust model; the existence of VSGs is probably a general characteristic of disks in normal galaxies.

\subsection{Big grains}

Table 5 shows that the bulk of the observed galaxies has a relatively cold dust component with temperatures $\simeq 20-23 \mathrm{~K}$. These temperatures agree with those obtained recently from Bianchi et al. (1999a) for a sample of seven spiral normal galaxies. To check if the FIR flux ratios trace correctly the temperatures of the cold dust we plot them in Figs. $7 \mathrm{a}-\mathrm{d}$ as a function of the $60 / 100,100 / 175,120 / 175$ and $100 / 200 \mu \mathrm{m}$ flux ratios respectively. The FIR ratios are independent from the assumed model. Therefore, if the fitted temperatures have some physical meaning, there should be a correlation between them and the FIR ratios. Figure 7 clearly shows that the fitted temperatures correlate with all the IR ratios but the $60 / 100 \mu \mathrm{m}$, the best correlation being that with the $100 / 200 \mu \mathrm{m}$ color ratio (Fig. 7d). This suggests that the FIR 60/100 ratio cannot be used to trace the cold dust temperature. This is probably due to a non negligible contribution of the VSGs to the $60 \mu \mathrm{m}$ emission. Mayya \& Rengarajan (1997) 

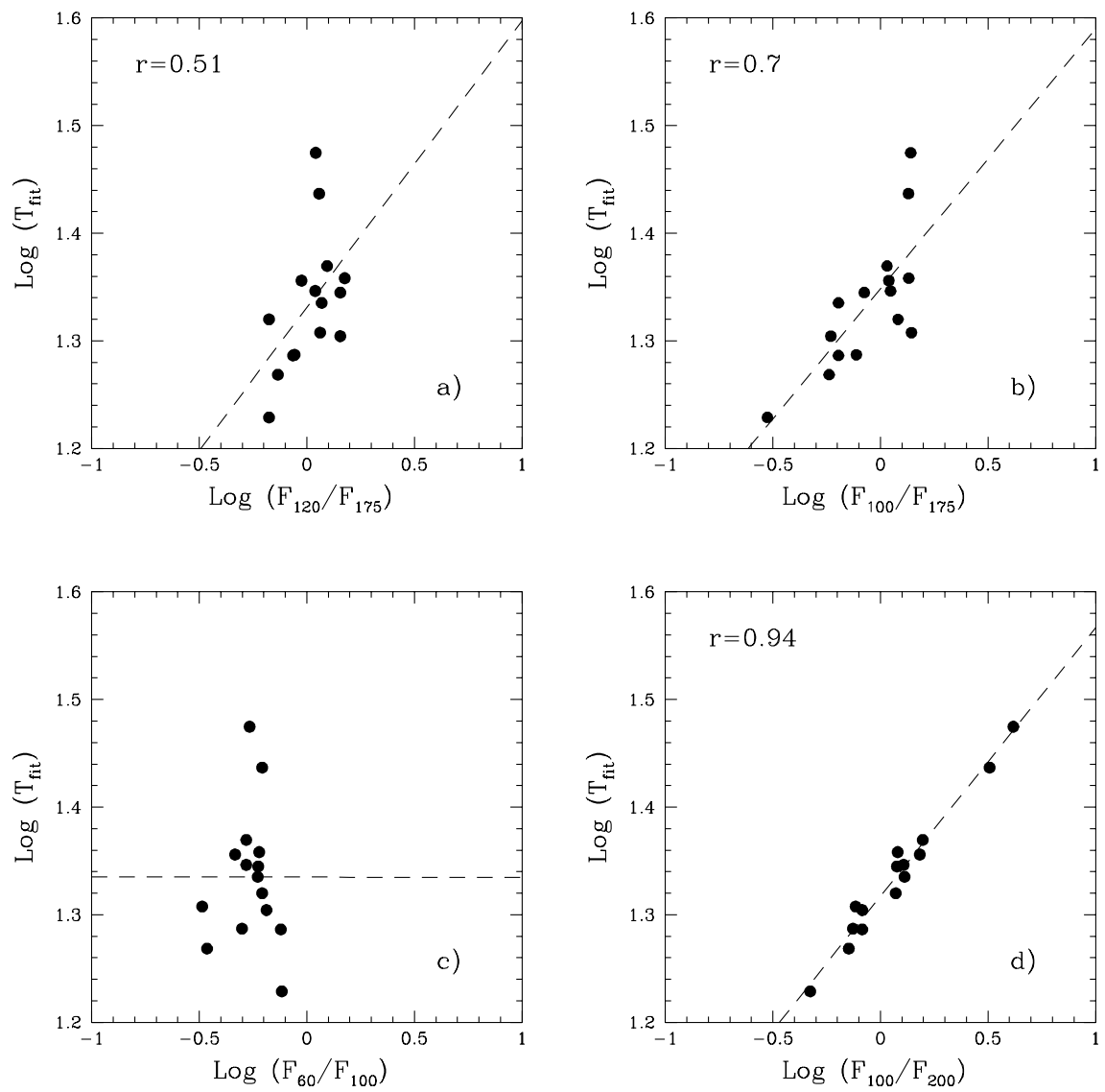

Fig. 7. The fitted temperatures as a function of the FIR color temperatures. The correlation coefficients $r$ are given in the upper-left of each plot

Table 6. The $15 / 6.75 \mu \mathrm{m}$ ratios of HII regions in galaxies CGCG 160095 and CGCG 97087 labeled in Figs. 3a and 3b

\begin{tabular}{lc}
\hline CGCG 160095 & $15 / 6.75 \mu \mathrm{m}$ \\
\hline HII 1 & 1.00 \\
HII 2 & 0.96 \\
HII 3 & 0.96 \\
Nucl. & 0.70 \\
\hline CGCG 97087 & $15 / 6.75 \mu \mathrm{m}$ \\
\hline $\mathrm{C}_{1}+\mathrm{C}_{2}$ & 1.50 \\
$\mathrm{~B}_{3}$ & 1.31 \\
\hline
\end{tabular}

showed that VSGs can contribute up to $60 \%$ of the flux at $60 \mu \mathrm{m}$ beyond half the disk radius in spiral galaxies. For these reasons we expect the infrared flux beyond $100 \mu \mathrm{m}$ being a better tracer of the BGs emission. Figure 8 shows the relationships between the infrared flux at 200, 175, 120, 100 and $60 \mu \mathrm{m}$ normalized to the $H$ band flux as function of the $\mathrm{H} \alpha E . W$. The correlation coefficients obtained show that the poorer correlation is that with the $200 \mu \mathrm{m}$ flux, the others being comparable. The fitted slopes decreases as the wavelength increases, as shown in Fig. 9.

Thus, as expected, the contribution of the youngest stellar population to the dust heating becomes less important towards longer infrared wavelengths.

\subsection{The dust-to-gas ratio}

A determination of the dust to gas ratio, as that derived in the solar neighborhood, is not straightforward since it implies the knowledge of the dust and gas spatial distributions in the target galaxies. In the solar neighborhood the dust to gas ratio is determined from dust and gas column densities (Sodrosky et al. 1994). For the present sample we have only total gas and dust masses (since they are unresolved by ISO). It is reasonable to assume that the cold dust is as extended as the optical disks, and that the HI disk is $\sim 1.8$ times larger than the optical one (and similar to the optical for the HI deficient objects) (Cayatte et al. 1994). In these assumptions we can estimate a value of the dust to gas ratio which is directly comparable to that observed in the solar neighborhood (Col. 5 in Table 5). Excluding 2 objects with a poor $M_{\text {dust }}$ estimate because of a bad quality fit, we obtain an average dust to gas ratio of $4.6( \pm 2.4) 10^{-3}$, a value consistent with the solar neighborhood one of $6.2510^{-3}$ (Sodrosky et al. 1994).

Figures 10a,b show the relationships between the dust masses and the atomic and molecular gas masses respectively.

Masses have been normalized to the luminosity in the $H$ band to remove the scaling effects. The cold dust mass of the target galaxies is only weakly related to the 

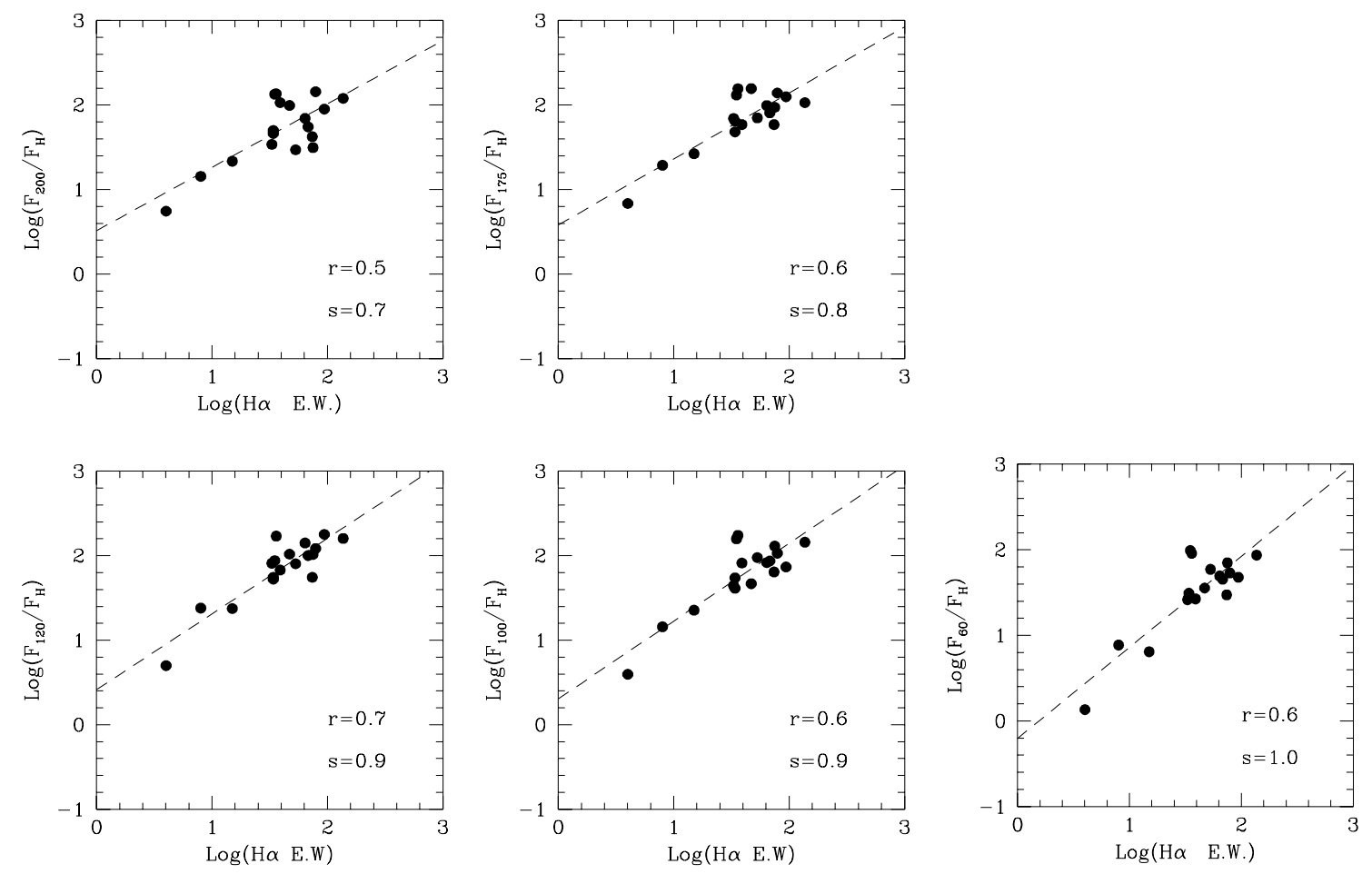

Fig. 8. $F_{200}, F_{175}, F_{120}, F_{100}, F_{60}$ normalized flux as function of $\mathrm{H} \alpha E . W$. in $\AA$ and the regression lines

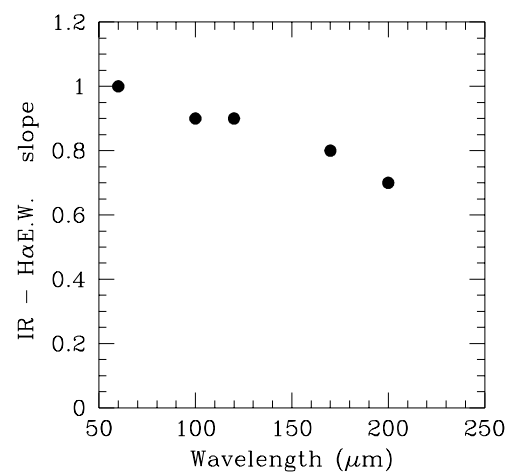

Fig. 9. The slopes of the regression lines obtained fitting the IR emission as function of the $\mathrm{H} \alpha E . W$. as a function of wavelength

molecular or atomic gas content, in agreement with $\mathrm{Xu}$ et al. (1997), suggesting that big dust grains are associated with both gas phases. This observational result, which should be confirmed with larger samples, does not confirm the strong $M_{\text {dust }}-M_{\mathrm{H}_{2}}$ relationship claimed by Young et al. (1989) and Lavezzi et al. (1998) based on dust masses determined from IRAS data, because their 60/100 flux ratio is an unreliable cold dust temperature estimator (see Sect. 5.3).

\subsection{Environmental effects}

The MIR emission properties of our sample of galaxies cluster do not differ from the typical MIR emission properties of field normal galaxies in agreement with other ISO results (Boselli et al. 1998). Nevertheless, some of the sampled galaxies are HI deficient (see Table 2). This parameter is defined as the ratio between the HI content of a cluster and a field galaxy of similar type $\mathrm{T}$ and size D (Giovanelli \& Haynes 1985). The HI reservoir of galaxies with HI def $>0.3$ has efficiently been stripped by the ram pressure exerted by the IGM.

The asymmetric HI distribution of the other galaxies of the sample, that would have been removed by the galaxy revolution on time scales of $\sim 10^{8}$ years, indicates that ram pressure is still an ongoing process (Gavazzi et al. 1995b). In these galaxies we are probably witnessing the beginning of the stripping process, whose final product will be a complete HI depletion.

As discussed in the introduction, the cold dust is expected to be more extended than the warm dust (Israel et al. 1999; Bianchi et al. 1999a; Krugel et al. 1998). We have shown in Sect. 5.3 that most of the analyzed galaxies have a cold component, even though we do not have any information about its spatial distribution and extension. It would not be surprising that the ram-pressure would be able to strip some cold dust from the outer parts of the galaxy disks.

However from the present sample we found that HIdeficient galaxies have on average dust masses comparable with those of HI-normal objects (Fig. 10a), as observed by Bicay \& Giovanelli (1987). This result seems however in disagreement with Doyon \& Joseph (1989), who found a deficiency in the dust content in late-type galaxies close to the Virgo cluster core. The lack of a well suited reference sample of isolated galaxies at wavelengths longer than $100 \mu \mathrm{m}$ prevents us from further investigating this issue. Moreover, the PHOT instrument could have not been sensitive enough to detect some of the external cold dust with 

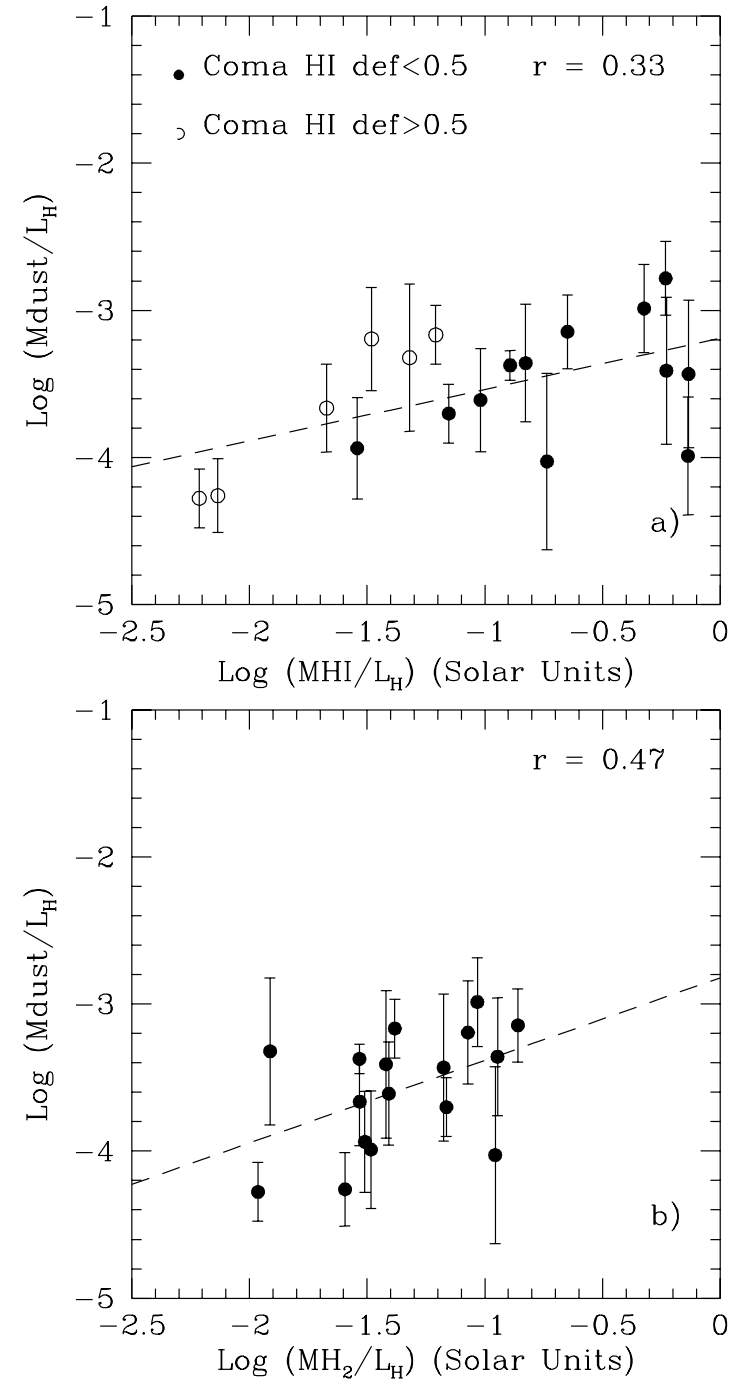

Fig. 10. The logarithmic plot of the dust mass versus the HI and $\mathrm{H}_{2}$ masses

low infrared surface brightness. This might result in underestimating the total dust mass. A conclusive analysis of this issue is pending on future infrared and sub-millimeter missions, like SIRTF and FIRST.

\section{Conclusions and summary}

ISOCAM and ISOPHOT observations of 18 late-type galaxies belonging to the Coma and A1367 clusters are presented in this paper. Complementary UV, Optical, NIR and $\mathrm{H} \alpha$ images and $\mathrm{HI}$ and $\mathrm{CO}$ line measurements made it possible to investigate the nature of the various dust components and to study the relationships between the dust and the star formation properties and between the dust and the gas (atomic and molecular) content.

Our mains conclusions are the following:

- The excitation of the AIB carriers responsible for the MIR emission between 5 and $20 \mu \mathrm{m}$ is a complex phenomenon: in some cases visible photons of the general ISRF are sufficient to produce extended MIR emission throughout galaxy disks far from the sites of massive star formation. The 6.75 and $15 \mu \mathrm{m}$ properties and the study of the $15 / 6.75 \mu \mathrm{m}$ ratios suggest that the total MIR spectra of the present galaxies are similar to those of PDR regions, i.e. spectra dominated by AIBs and with relatively flat continua. On the other hand, strong UV radiation fields produced in star formation regions provide the principal source of excitation of local AIB carriers, until the UV radiation field associated with them is strong and hard enough to destroy the AIB carriers. This explains the different MIR and $\mathrm{H} \alpha$ surface brightness distributions;

- A warm dust component dominating the emission in the range $25-60 \mu \mathrm{m}$ is likely to exist in the observed galaxies. It could arise from VSGs and/or hot BGs. Only close to active HII regions the VSGs are heated to such temperatures that their emission becomes important at $15 \mu \mathrm{m}$, thus increasing the $15 / 6.75$ ratio;

- A cold BG dust component peaking at $\simeq 100 \mu \mathrm{m}$ is present in all galaxies with temperatures $\sim 20-23 \mathrm{~K}$. With the present data, we cannot rule out the existence of an even colder dust component;

- The contribution of the youngest stellar population to the cold dust heating becomes smaller going towards long infrared wavelengths;

- The dust to gas ratio of the observed galaxies is comparable to that of the solar neighborhood;

- The weak observed trend between the total dust mass and the molecular and atomic gas content suggests that the cold dust is associated to both gas phases.

Finally, we do not observe any significant difference in the IR emission properties of the present galaxy sample in respect to the IR emission properties observed in normal field galaxies, despite the fact that our sample is dominated by galaxies with disk star formation activities higher than the corresponding galaxies in the field. HI-deficient galaxies have dust masses comparable to unperturbed objects, suggesting that the mechanism responsible for the neutral gas stripping is not able to strip the relatively cold dust.

Acknowledgements. A. Contursi wishes to thank Veronique Buat, Fabienne Casoli, Daniel Dale, George Helou, Susanne Madden, Gary Neugebauer, Marc Sauvage, and Nik Scoville, for useful discussions and the support of the IPAC institute.

\section{References}

Abergel, A., Bernard, J., Boulanger, F., et al. 1996, A\&A, 315, L329

Allamandola, L. J., Tielens, G., \& Barker, J. 1989, ApJS, 71,733

Allamandola, L. J., Hudgins, D. M., \& Sandford, S. A. 1999, ApJ, 511, 115

Alton, P. B., Xilouris, E. M., Bianchi, S., et al. 2000, A\&A, 356,795

Beichman, C. A. 1987, ARA\&A, 24, 521

Bianchi, S., Alton, P. B., \& Davies, J. I. 1999a, in Proc. of ISO Beyond Point Sources: Studies of Extended Infrared Emission, ed. R. J. Laureijs, K. Leech, \& M. F. Kessler, ESA-SP 455, 2000, p. 149 [astro-ph/9910011] 
Bianchi, S., Davies, J. I., \& Alton, P. B. 1999b, A\&A, 344, L1

Bicay, M., \& Giovanelli, R. 1987, ApJ, 321, 645

Boselli, A., Lequeux, J., Contursi, A., et al. 1997, A\&A, 324, L13

Boselli, A., Gavazzi, G., Lequeux, J., et al. 1997, A\&A, 327, 522

Boselli, A., Lequeux, J., Sauvage, M., et al. 1998, A\&A, 335, 53

Boulade, O., Sauvage, M., Altieri, B., et al. 1996, A\&A, $315, \mathrm{~L} 85$

Boulanger, F., Beichman, C., Désert, F., et al. 1988, ApJ, 332,328

Boulanger, F., Reach, W. T., Abergel, A., et al. 1996, A\&A, 315, L329

Cayatte, V., Kotanyi, C., Balkowski, C., van Gorkom, J. 1994, AJ, 107, 1003

Cesarsky, D., Lequeux, J., Abergel, A., et al. 1996a, A\&A, 315, L305

Cesarsky, C. J., Abergel, A., Agnèse, P., et al. 1996, A\&A, 315, L32

Cesarsky, D., Lequeux, J., Abergel, A., et al. 1996b, A\&A, 315, L309

Contursi, A., Lequeux, J., Hanus, M., et al. 1998, A\&A, 336,662

Contursi, A., Lequeux, J., Cesarsky, D., et al., accepted to A\&A [astro-ph/0006185]

Dale, D. A., Helou, G., Silbermann, N., et al. 1999, AJ, 118,2055

Dale, D. A., Silbermann, N., Helou, G., et al. 2000, AJ [astro-ph/0005083]

Désert, F. X., Boulanger, F., \& Puget, J. L. 1990, A\&A, 237,215

Devereux, N. A., \& Young, J. S. 1990, ApJ, 350, L25

Devereux, N. A., \& Young, J. S. 1993, AJ, 106, 2587

Devereux, N. A., \& Scowen, P. A. 1994, AJ, 108, 1667

Donas, J., Milliard, B., Laget, M., \& Buat, V. 1990, A\&A, 235,60

Donas, J., Milliard, B., \& Laget, M. 1995, A\&A, 303, 661

Doyon, R., \& Joseph, R. D. 1989, MNRAS, 239, 347

Draine, B. T, \& Lee, H. M. 1984, ApJ, 285, 89

Draine, B. T, \& Anderson, N. 1985, ApJ, 292, 494

Gabriel, C., Acosta-Pulido, J., \& Heinrichsen, I. 1997, ADASS VI, ASP Conf. Ser., vol. 125, ed. G. Hunt, \& H. E. Payne, 108.

Gavazzi, G., Tarenghi, M., Jaffe, W., et al. 1984, A\&A, 137, 235

Gavazzi, G. 1989, ApJ, 346, 59

Gavazzi, G., Randone, I., \& Branchini, E. 1995a, ApJ, 438, 590

Gavazzi, G., Contursi, A., Carrasco, L., et al. 1995b, A\&A, 304,325

Gavazzi, G., \& Boselli, A. 1996, Astroph. Lett. \& Commun., 35,1

Gavazzi, G., Pierini, D., \& Boselli, A. 1996, A\&A, 312, 397

Gavazzi, G., Catinella, B., Carrasco, L., et al. 1998, AJ, 115,1745
Giovanelli, R., \& Haynes, M. 1985, ApJ, 292, 404

Helou, G. 1986, ApJ, 311, L33

Helou, G., Lu, N. Y., Werner, M. W., et al. 2000, ApJ, in press

Israel, F. P., van der Werf, P. P., \& Tilanus, R. P. J. 1999, A\&A, 344, L83

Laurent, O., Mirabel, I. F., Charmandaris, V., et al. 1999, in The Universe as seen by ISO, ed. P. Cox, \& M. K. Kessler, ESA SP-427, France, vol. 2, 913

Lavezzi, T. E., Dickey, J., Casoli, F., \& Kazès, I. 1999, AJ, 117,1995

Le Coupanec, P., Rouan, D., Gallais, P., et al. 1999, in The Universe as seen by ISO, ed. P. Cox, \& M. K. Kessler, ESA SP-427, France, vol. 2, 917

Léger, A., \& Puget, J. L. 1984, A\&A, 137, L5

Lemke, D., Klaas, U., Abolins, J., et al. 1996, A\&A, 315, L64

Kessler, M. F., Steinz, J. A., Anderegg, M. E., et al. 1996, A\&A, 315, L27

Krugel, E., Siebenmorgen, V., Zota, V., et al. 1998, A\&A, 331, L9

Mathis, J. S. 1990, ARA\&A, 28, 37

Mayya, Y. D., \& Regaradjan, T. N. 1997, AJ, 114, 946

Mirabel, I. F., Vigroux, L., Charmandaris, V., et al. 1998, A\&A, 333, L1

Nilson, P. 1973, Uppsala Obs. Ann, 6 (UGC)

Pagani, L., Lequeux, J., Cesarsky, D., et al. 1999, A\&A, 351, 447

Persson, C. J., Lonsdale, \& Helou, G. 1987, ApJ, 314, 513

Puget, J. L., \& Léger, A. 1989, ARA\&A, 27, 161

Rice, W., Boulanger, F., Viallefond, F., et al. 1990, ApJ, 358,418

Sauvage, M., \& Thuan, T. X. 1992, ApJ, 396, L69

Schubert, J., Fouks, B. I., Lemke, D., \& Wolf, J. 1995, Proc SPIE, 2553, 461

Sodrosky, T. J., Bennett, C., Boggess, N., et al. 1994, ApJ, 428,638

Soifer, B. T., Houck, J. R., \& Neugebauer, G. 1987, ARA\&A, 25,187

Starck, J. L., Abergel, A., \& Aussel, H. 1999, A\&AS, 134, 135

Sullivan, W., Bothun, G., Bated, B., et al. 1981, AJ, 86, 91

Uchida, K. J., Sellgren, K., \& Werner, M. 1998, ApJ, 493, L109

Vigroux, L., Charmandaris, V., \& Gallais, P. 1999, in The Universe as seen by ISO, ed. P. Cox, \& M. K. Kessler, ESA SP-427, France, vol. 2, 805

Young, J., Xie, S., Kenney, J. D. P., et al. 1989, ApJS, 70, 699

Xu, C., \& Helou, G. 1996, ApJ, 456, 152

Xu, C., Buat, V., Boselli, A., \& Gavazzi, G. 1997, A\&A, 324, 32

Walterbos, R. A. M., \& Schwering, P. B. W. 1987, A\&A, 180, 27

Zwicky, F., \& Kowal, C. T. 1961-1968, Catalogue of Galaxies and Clusters of Galaxies, vol. 2 (Pasadena: Caltech) (GCGC) 